\title{
复合工况下四轮驱动电动汽车操纵稳定性控制"
}

\author{
许男李小雨 \\ (吉林大学汽车仿真与控制国家重点实验室 长春 130022)
}

\begin{abstract}
摘要: 设计一个考虑车辆侧纵向耦合的模型预测控制器(Model predictive control, MPC)用于提升四轮驱动电动汽车在复合工况 下的操纵性和稳定性。基于传统 UniTire 轮胎模型, 推导面向控制器设计的复合工况 UniTire 轮胎模型解析表达式; 基于前后 轮侧偏角相平面提出一种新的车辆稳定性评价标准, 通过前后轮侧偏角的位置到坐标原点的距离判断车辆的稳定性, 给出定 量的稳定性指标; 采用 MPC 控制器结构将车辆操纵性控制、稳定性控制以及四轮滑移率约束作为目标函数, 采用复合工况 UniTire 轮胎模型表达式和双轨车辆模型构建预测模型, 并且通过稳定指标和轮胎滑移率实时调整各个目标的权重以达到最优 的控制效果; 通过 Simulink-CarSim 联合仿真和硬件在环(HiL)测试对算法进行验证, 结果表明, 该控制器能够根据车辆稳定 性指标动态调节跟踪权重, 有效提升车辆的操纵性和稳定性。并且由于复合 UniTire 轮䏩模型的使用, 极限工况下控制器决 策的四轮力矩更小, 有效地抑制轮胎滑移率的增加。
\end{abstract}

关键词: 四轮驱动电动汽车; 复合工况 UniTire 轮胎模型; 稳定性指标; 模型预测控制; 轮胎侧偏角相平面; 硬件在环测试 中图分类号: U461

\section{Handling and Stability Control of Four-wheel Drive Electric Vehicle under Combined Slip Conditions}

\author{
XU Nan LI Xiaoyu
}

(State Key Laboratory of Automotive Simulation and Control, Jilin University, Changchun 130022)

\begin{abstract}
A model predictive controller(MPC) considering combined slip conditions is designed to improve the handling and stability of four-wheel drive electric vehicles. Firstly, the UniTire model is simplified and deduced, and the analytical expressions of the combined UniTire model which can be used for the controller design are obtained. Secondly, based on the analysis of the tire slip angle phase plane, a new vehicle stability evaluation standard is proposed. The vehicle stability can be judged by the distance from the position of the front and rear tire slip angles to the coordinate origin, and the quantitative stability index can be obtained. Then, the MPC structure is used to take the vehicle handling control, stability control and four-wheel slip ratio constrains as the objective function. Combined UniTire model and the double-track vehicle model are used as the prediction model, and the weight of each objective is adjusted in real time through the stability index and tire slip ratio to achieve the optimal control effect. Finally, the algorithm is verified by Simulink-CarSim simulation and hardware in the loop(HiL) test. The results show that the controller can dynamically adjust the tracking weight according to the vehicle stability index, and improve the handling and stability of the vehicle. Due to the application of the combined UniTire model, the four-wheel torque decided by the controller under the harsh condition is smaller, which effectively inhibits the increase of tire slip ratio.
\end{abstract}

Key words: four-wheel drive electric vehicle; combined UniTire model; stability index; model predictive control; tire slip angle phase plane; hardware in the loop test

\section{0 前言}

电动汽车有利于减少环境污染降低化石能源消

\footnotetext{
* 国家自然科学基金(61790561，51875236)和中国汽车产业创新发展联 合基金(U1864206)资助项目。20200203 收到初稿, 20210125 收到修改 稿
}

耗, 因而成为汽车行业发展的重要方向, 受到各国 的重视 ${ }^{[1]}$ 。其中四轮驱动电动汽车由于具有更加简 单的动力传动系统以及更加灵活的驱动方式, 而受 到更多的关注 ${ }^{[2]}$ 。与传统燃油车(Internal combustion engine vehicles, ICVs)相比, 四轮驱动电动汽车的四 轮力矩和角速度能够更加精准地实现独立控制, 因 
而为提高车辆安全性和操纵性提供了完美的研究平 台 ${ }^{[3]}$ 。

当前, 已有很多关于提升车辆操纵性的研究。 文献[4-5]通过跟踪线性二自由度模型的横摆运动来 提升车辆的操纵性, 文献[6]通过允许质心侧偏角达 到更大的值来提升车辆在转向时的机动性能, 文 献[7]分析了主动横摆力矩对车辆转向特性的影响, 从而提出了考虑瞬态响应的横摆运动期望值来提升 车辆操纵性能。尽管这些方法能够有效地提升车辆 操纵性, 但是这些方法并没有考虑可靠的安全约束, 极限工况下车辆很可能会失稳。

为了提升车辆的稳定性, 相平面方法作为一种 图形化的稳定性判断方法被广泛采用。文献[8]利用 质心侧偏角-质心侧偏角变化率 $(\beta-\dot{\beta})$ 相平面确定 的稳定区域用于设计车辆电子稳定系统。文献[9]采 用同样的稳定边界用于四轮驱动电动汽车的稳定性 和节能控制。文献[10]采用前后轮侧偏角 $\left(\alpha_{f}-\alpha_{r}\right)$ 构成的相平面作为稳定性约束, 采用模型预测控制 方法(Model predictive control, MPC)提升车辆的稳 定性。文献[11-12]通过在质心侧偏角-横摆角速度 $(\beta-r)$ 相平面中设计稳定包络区域, 通过包络控制 的方法使车辆状态处于安全区域, 从而提升车辆的 稳定性。文献[13]采用同样的包络区域作为自动驾 驶汽车的稳定性约束。尽管上述基于相平面的方法 能够在一定程度上提升车辆的稳定性, 但是它们也 存在明显的不足。由于相平面稳定性边界划分受到 多种因素的影响(模型简化、车辆参数以及路面摩擦 因数的不确定性), 不准确的信息将会使决策的边界 与实际值出现较大的偏差, 从而导致控制器不能及 时动作进而使车辆失稳 ${ }^{[14]}$ 。此外, 这些方法多采用 线段对二维相平面进行区域分割确定稳定和不稳定 区域, 有可能导致车辆状态在稳定边界上来回抖动, 影响控制效果 ${ }^{[10-11]}$ 。为此本文的第一个目标是提出 一种更加先进的车辆稳定性判断方法, 通过车辆前 后轮胎侧偏角状态确定车辆的稳定裕度, 给出定量 的稳定性指标。

当前, 为了降低控制器的复杂度车辆稳定性控 制器多采用纯侧偏/纵滑 ${ }^{[15]}$ 或线性轮胎模型 ${ }^{[10]}$ 。不考 虑纵向力与侧向力之间的耦合作用。但是在极限工 况下, 控制器需要决策主动横摆力矩以达到车辆的 稳定性控制效果。此时纵向力(绝对值)会明显增大, 导致侧向力的衰减, 采用纯侧偏/纵滑轮胎模型就不 足以反映此时的轮胎特性, 影响控制效果 ${ }^{[16]}$ 。四 轮驱动电动汽车力矩响应快而且四轮力矩独立可 控, 因而更容易受到侧纵向力耦合作用的影响。由
郭孔辉 ${ }^{[17]}$ 提出的 UniTire 轮胎模型能够准确地描述 纯侧偏/纵滑以及复合工况下轮胎力学特性。为了提 升四轮驱动电动汽车在极限工况下控制力矩的分配 效果, 本文将对 UniTire 轮胎模型进行简化和推导, 以使其能够满足控制器设计的要求, 提升车辆控制 效果。

随着技术的发展, 对运算能力有较高要求的模 型预测控制算法(Model predictive control, MPC)也 开始被应用于车辆的控制 ${ }^{[18-20]}$ 。MPC 能够通过数学 模型预测一段时间(预测时域)内车辆的行为, 有利 于控制器决策出更加合理地控制输出。而且 MPC 控制器结构能够将多种目标约束考虑到控制器中进 行多目标控制, 求解出符合执行器约束的最优控制 结果。因此, 本文采用 MPC 方法进行控制器设计, 控制目标包括：操纵性控制、稳定性控制以及四轮 滑移率约束。然后将这些控制目标集成到 MPC 控 制器中, 通过车辆状态以及轮胎滑移率对各个控制 目标的权重进行调节, 从而实现复合工况下四轮驱 动电动汽车的最优控制。

最后本文通过 Simulink-CarSim 软件平台对控 制算法的效果进行了仿真验证, 并采用硬件在环测 试了算法在工程应用中的实时性。

\section{1 轮胎模型}

\section{1 面向车辆动力学仿真的 UniTire 轮胎模型}

用于车辆动力学仿真的轮胎模型精度决定了仿 真结果的准确程度。本研究采用 UniTire 轮胎模型 作为仿真模型 ${ }^{[21]}$ 。UniTire 轮胎模型是非线性半经验 轮胎模型, 在获得轮胎辨识参数后, 能够准确地描 述复合工况轮胎的非线性特性 ${ }^{[17]}$ 。模型描述如下。

轮胎纵向滑移率 $S_{x}$ 和侧向滑移率 $S_{y}$ 表示为

$$
\left\{\begin{array}{l}
S_{x}=\frac{\omega R_{e}-v_{x}}{\omega R_{e}} \\
S_{y}=\frac{-v_{y}}{\omega R_{e}}
\end{array}\right.
$$

式中, $\omega$ 是车轮的旋转角速度, $R_{e}$ 为有效滚动半径; $v_{x}$ 和 $v_{y}$ 分别为车轮运动速度 $v$ 在纵向和侧向上的 分量。通常, 纵向滑移率也被定义为 $k=\left(\omega R_{e}-v_{x}\right) / v_{x}$, 因此 $S_{x}$ 与 $\kappa$ 之间的关系为 $S_{x}=\kappa /(1+\kappa)^{[22]}$ 。

相对纵向、侧向和综合滑移率 $\phi_{x} 、 \phi_{y}$ 和 $\phi$ 的定 义为

$$
\phi_{x}=\frac{K_{x} S_{x}}{\mu_{x} F_{Z}} \quad \phi_{y}=\frac{K_{y} S_{y}}{\mu_{y} F_{Z}} \quad \phi=\sqrt{\phi_{x}^{2}+\phi_{y}^{2}}
$$


式中, $K_{x}$ 和 $K_{y}$ 分别是轮胎的纵滑和侧偏刚度; $\mu_{x}$ 和 $\mu_{y}$ 分别是轮胎与路面间的纵向和侧向摩擦因数, $F_{Z}$ 表示垂向载荷。

UniTire 轮胎模型可以描述为

$$
\left\{\begin{array}{l}
\bar{F}=1-\exp \left[-\phi-E \phi^{2}-\left(E^{2}+\frac{1}{12}\right) \phi^{3}\right] \\
F_{x}=\mu_{x} \frac{\phi_{x}}{\phi} F_{z} \bar{F} \\
F_{y}=\mu_{y} \frac{\phi_{y}}{\phi} F_{z} \bar{F}
\end{array}\right.
$$

式中, $\bar{F}$ 表示无量纲总切力, $F_{x}$ 为轮胎纵向力, $F_{y}$ 为轮胎侧向力, $E$ 为曲率因数, 模型中参数的具体 表达式见文献[23]。

复合工况下考虑方向因子 $\lambda$ 有利于提高轮胎模 型的表达精度 ${ }^{[24]}$, 方向因子

$$
\lambda=\frac{1+\left(\phi / \phi_{\mathrm{c}}\right)^{n} \cdot K_{y} / K_{x}}{1+\left(\phi / \phi_{\mathrm{c}}\right)^{n}}
$$

式中, $\phi_{c}$ 和 $n$ 为控制参数。

由于方向因子 $\lambda$ 的引入, 可得新的相对纵向、 侧向以及综合滑移率

$$
\phi_{x n}=\lambda \phi_{x} \quad \phi_{y n}=\phi_{y} \quad \phi_{n}=\sqrt{\phi_{x n}^{2}+\phi_{y n}^{2}}
$$

因此, 新的轮胎纵向力和侧向力分别为

$$
\left\{\begin{array}{l}
F_{x}=\mu_{x} \frac{\phi_{x n}}{\phi_{n}} F_{z} \bar{F} \\
F_{y}=\mu_{y} \frac{\phi_{y n}}{\phi_{n}} F_{z} \bar{F}
\end{array}\right.
$$

然后, UniTire 轮胎模型与 Pirelli 205/40R 18 轮 胎试验数据进行了对比验证(轮胎测试设备为 MTS Flat-Trac plus 高速轮胎试验台, 模拟路面摩擦因数 约为 1.1)。结果如图 1 4 所示, UniTire 轮胎模型 能够很好地描述纯工况和复合工况下的轮胎力学特 性。而且 UniTire 模型具有 “plug-in” 摩擦因数的特 点 ${ }^{[17]}$, 能够适用不同的路面摩擦条件, 除摩擦因数 外, 不需要调整其他轮胎模型参数。

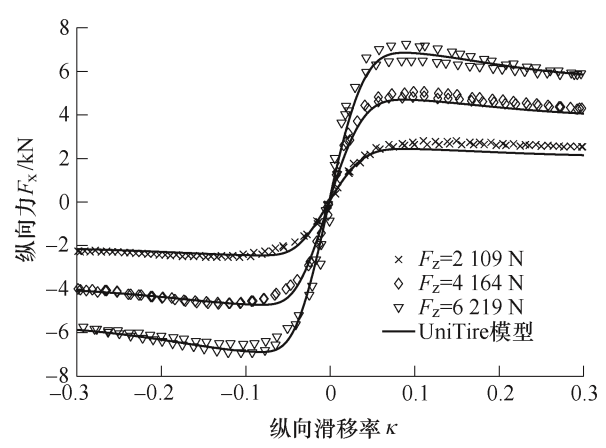

图 1 UniTire 模型对比试验数据: 纯纵滑工况

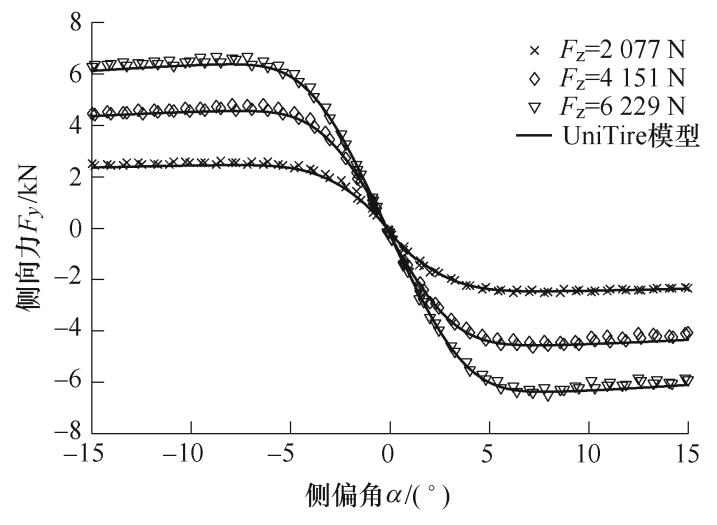

图 2 UniTire 模型对比试验数据: 纯侧偏工况

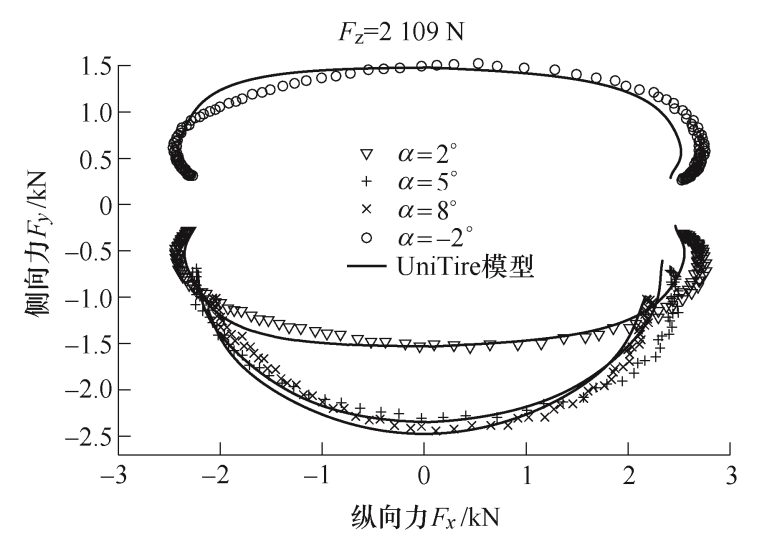

图 3 UniTire 模型对比试验数据: 复合工况, $F_{z}=2109 \mathrm{~N}$

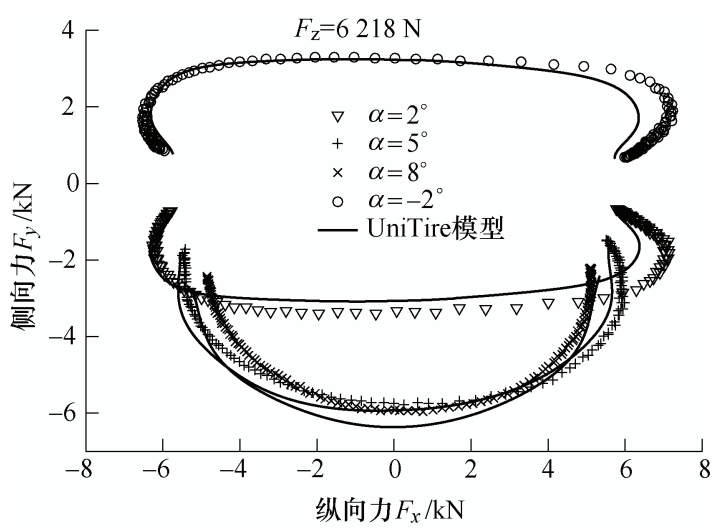

图 4 UniTire 模型对比试验数据: 复合工况, $F_{z}=6218 \mathrm{~N}$

\section{2 面向控制的复合工况 UniTire 轮胎模型}

复合工况轮胎模型能够更加准确地描述轮胎纵 向力对侧偏特性影响, 用于控制器设计能够提升车 辆在复杂工况下的控制效果 ${ }^{[16]}$ 。这是因为复合工况 条件下轮胎力学特性特别是侧偏特性会发生明显的 变化, 对车辆的稳定性产生不利影响。准确地描述 复合工况下轮胎侧偏特性的变化规律是提升复合工 况下车辆稳定性控制的先决条件。

为了准确描述轮胎力学特性的耦合和非线性特性, 当前的轮胎模型常采用复杂的表达形式, 以提高车辆 动力学的仿真精度。然而复杂的轮胎模型结构难于直 接应用于常规的控制器设计, 虽然非线性控制器能够 
将轮胎模型代入到控制器结构中 ${ }^{[25]}$, 但是会导致控 制系统结构复杂, 运算量增大, 难以在实际工程中 应用。

因而本节将建立面向控制的 UniTire 轮胎模型, 保留原 UniTire 模型对复合工况的表达能力, 将轮 胎力学的侧纵向耦合和非线性特征集中在有效侧 偏刚度的表达中, 能够非常方便地进行控制器设计, 将原来复杂的非线性控制器设计问题转化为线性时 变问题, 大大简化了控制器设计方案, 提高优化求 解速度。具体推导过程如下。

由式(6)可得

$$
F_{y}=\frac{\bar{F}}{\phi_{n}} \mu_{y} F_{z} \frac{K_{y}}{\mu_{y} F_{z}} S_{y}
$$

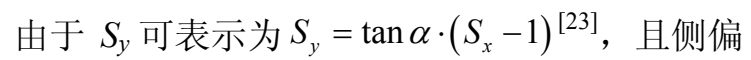
角 $\alpha$ 较小时有 $\tan \alpha \approx \alpha$, 因而式(7)可改写为

$$
F_{y} \approx K_{y c} \alpha
$$

式中, $K_{y c}$ 为有效侧偏刚度, 可表示为

$$
K_{y c}=\frac{\bar{F}}{\phi_{n}} K_{y}\left(S_{x}-1\right)
$$

然后 $K_{y c}$ 的导数可表示为

$$
\begin{aligned}
& \dot{K}_{y c}=\frac{\mathrm{d}}{\mathrm{d} t}\left(\frac{\bar{F}}{\phi_{n}} K_{y}\left(S_{x}-1\right)\right)= \\
& \frac{\mathrm{d}}{\mathrm{d} S_{x}}\left(\frac{\bar{F}}{\phi_{n}} K_{y}\left(S_{x}-1\right)\right) \cdot \frac{\mathrm{d} S_{x}}{\mathrm{~d} t} \approx \tilde{K}_{y} \cdot \frac{\mathrm{d} S_{x}}{\mathrm{~d} t} \\
& \tilde{K}_{y}=K_{y} \cdot \frac{\left[\frac{\mathrm{d} \bar{F}}{\mathrm{~d} \phi} \cdot \frac{\mathrm{d} \phi}{\mathrm{d} S_{x}}\left(S_{x}-1\right)+\bar{F}\right] \phi_{n}-\frac{\mathrm{d} \phi_{n}}{\mathrm{~d} S_{x}} \bar{F}\left(S_{x}-1\right)}{\phi_{n}^{2}} \\
& \frac{\mathrm{d} \bar{F}}{\mathrm{~d} \phi}=\left[1+2 E \phi+3\left(E^{2}+1 / 12\right) \phi^{2}\right] \times \\
& \exp \left[-\phi-E \phi^{2}-\left(E^{2}+1 / 12\right) \phi^{3}\right] \\
& \frac{\mathrm{d} \phi}{\mathrm{d} S_{x}}=\frac{\mathrm{d} \phi}{\mathrm{d} \phi_{x}} \cdot \frac{\mathrm{d} \phi_{x}}{\mathrm{~d} S_{x}}=\frac{\phi_{x}}{\phi} \cdot \frac{K_{x}}{\mu_{x} F_{z}} \\
& \frac{\mathrm{d} \phi_{n}}{\mathrm{~d} S_{x}}=\frac{\mathrm{d} \phi_{n}}{\mathrm{~d} \phi_{x}} \cdot \frac{\mathrm{d} \phi_{x}}{\mathrm{~d} S_{x}}=\frac{\lambda \phi_{x}}{\phi_{n}}\left(\frac{\mathrm{d} \lambda}{\mathrm{d} \phi_{x}} \cdot \phi_{x}+\lambda\right) \frac{K_{x}}{\mu_{x} F_{z}} \\
& \frac{\mathrm{d} \lambda}{\mathrm{d} \phi_{x}}=\frac{\left(\frac{K_{c} n}{\phi_{c}^{n}} \phi^{n-1} \cdot \frac{\mathrm{d} \phi}{\mathrm{d} \phi_{x}}\right)\left(1+\left(\frac{\phi}{\phi_{c}}\right)^{n}\right)}{\left(1+\left(\frac{\phi}{\phi_{c}}\right)^{n}\right)^{2}}- \\
& \frac{\left(1+K_{c}\left(\frac{\phi}{\phi_{c}}\right)^{n}\right)\left(\frac{n}{\phi_{c}^{n}} \phi^{n-1} \cdot \frac{\mathrm{d} \phi}{\mathrm{d} \phi_{x}}\right)}{\left(1+\left(\frac{\phi}{\phi_{c}}\right)^{n}\right)^{2}}
\end{aligned}
$$

最后将上述推导的表达式与原 UniTire 复合工 况轮胎模型进行了对比验证(图 5 7)。图 5 中的实 线为原 UniTire 轮胎模型直接计算的侧向力结果, 虚线为 $K_{y c}$ 乘以侧偏角 $\alpha$ 得到的结果。在图 6 和图 7 中, 实线为原 UniTire 模型计算的侧向力 $F_{y}$ 除以侧 偏角 $\alpha$ 得到真实的有效侧偏刚度, 然后该真实的有 效侧偏刚度对 $S_{x}$ 进行数值微分得到。而虚线为 $\dot{K}_{y c}$ 表达式(10)直接计算的结果。

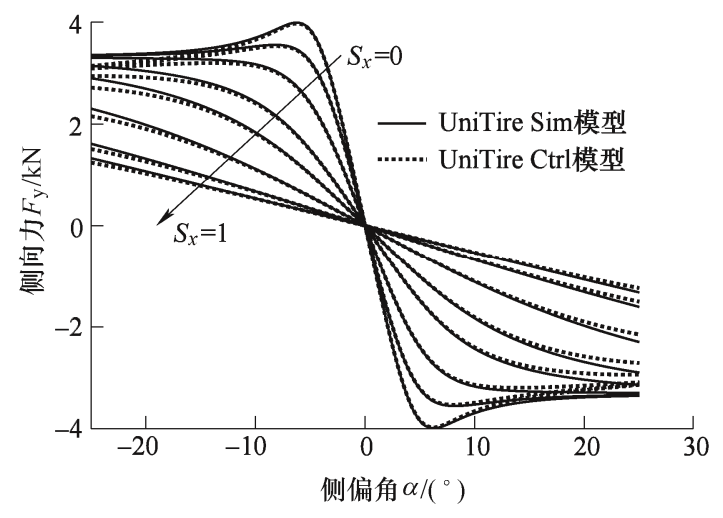

图 $5 K_{y c}$ 表达式对比 UniTire 模型

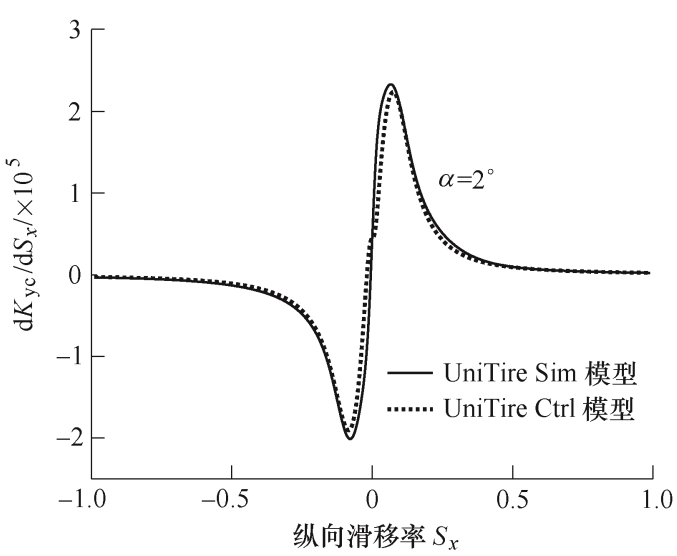

图 $6 \dot{K}_{y c}$ 表达式对比 UniTire 模型: $\alpha=2^{\circ}$

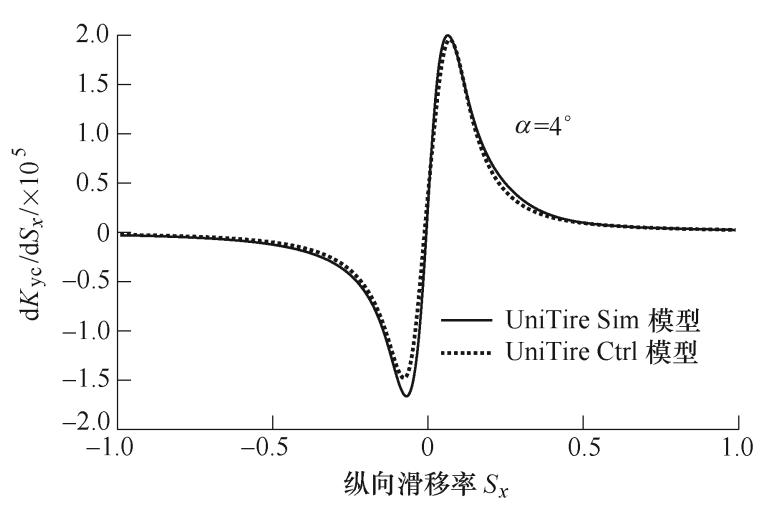

图 $7 \dot{K}_{y c}$ 表达式对比 UniTire 模型: $\alpha=4^{\circ}$

从图 5 可以看到简化后的表达式与原模型得 到的结果有很好的一致性，同时可以看到纵向滑 移率的增大会明显地降低轮胎有效侧偏刚度, 推 
导出的表达式也能够准确地描述有效侧偏刚度的 变化率。

\section{2 模型预测控制器(MPC)设计}

本文采用模型预测控制器对四轮驱动电动汽车 进行控制以提升其操纵稳定性。首先采用双轨车辆 模型和复合 UniTire 模型作为预测模型; 然后根据 线性二自由度模型得到理想车辆状态作为控制器的 参考状态; 接着在前后轮侧偏角相平面中对车辆稳 定性的变化规律进行了分析, 提出了定量的车辆稳 定性评价指标; 之后设计了模型预测控制器(MPC), 给出了考虑多目标约束的评价函数; 最后对系统约 束进行了设计。

\section{1 预测模型}

预测模型采用车辆平面运动双轨模型, 考虑四 个车轮的旋转自由度(图 8)。选择状态矢量为

$$
\boldsymbol{X}=\left(\begin{array}{llllllll}
\beta & r & F_{y f} & F_{y r} & e_{\omega f l} & e_{\omega f r} & e_{\omega r l} & e_{\omega r r}
\end{array}\right)^{\mathrm{T}}
$$

式中, $\beta$ 表示质心侧偏角, $r$ 为横摆角速度, $F_{y i}=F_{y i l}+F_{y i r}$ 表示第 $i$ 轴侧向力, $e_{\omega i j}$ 为车轮实际转速 与所期望转速的转速差。

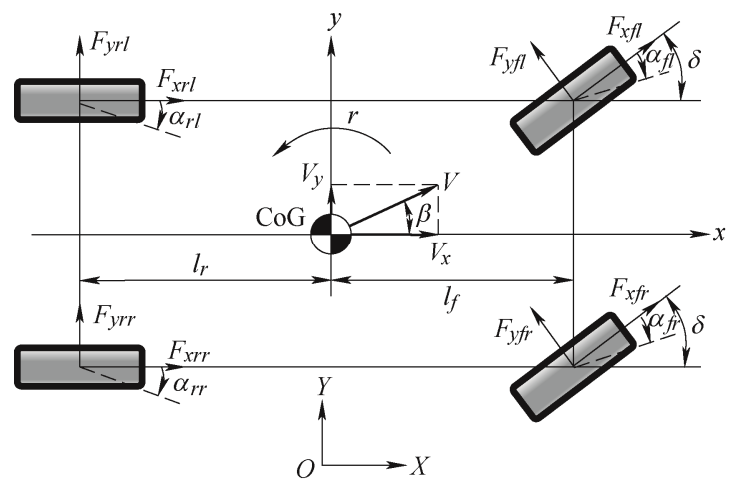

图 8 双轨车辆模型

文中所期望转速设定为轮胎纯滚动状态的转速 (详见第 2.4 节), 因而 $e_{\omega i j}$ 可用来表征轮胎的滑移状 态, 将该变量作为状态变量进行集中控制, 能够在 控制车辆操纵稳定性的同时, 控制车轮的滑移率, 防止车轮过度滑移。本文中下标 $i=f, r$ 分别表示前、 后; $j=l, r$ 分别表示左、右。

本文的研究对象是四轮驱动电动汽车, 四轮力 矩独立可控, 因而输入矢量

$$
\boldsymbol{U}=\left(\begin{array}{llll}
T_{q f l} & T_{q f r} & T_{q r l} & T_{q r r}
\end{array}\right)^{\mathrm{T}}
$$

式中, $T_{q i j}$ 为第 $i j$ 个车轮上驱动电机产生的力矩。

车辆的侧向动力学主要由质心侧偏角 $\beta$ 和横摆 角速度 $r$ 描述，可表示为

$$
\dot{\beta}=\frac{a_{y}}{u}-\frac{a_{x}}{u} \beta-r
$$

式中, $u$ 表示纵向速度 $\left(u=V_{x}\right)$, 侧向加速度

$$
a_{y}=\frac{1}{m}\left(F_{y f} \cos \delta+F_{x f} \sin \delta+F_{y r}\right)
$$

式中, $m$ 表示整车质量, $\delta$ 为前轮转角, $F_{x f}$ 为前轴 纵向力。

横摆角加速度可表示为

$$
\dot{r}=\frac{1}{I_{z}}\left(M_{F x}+M_{F y}\right)
$$

式中, $I_{z}$ 为车辆横摆转动惯量, $M_{F x}$ 与 $M_{F y}$ 分别为纵 向力和侧向力作用而产生的横摆力矩, 分别可表 示为

$$
\begin{gathered}
M_{F x}=F_{x f l}\left(l_{f} \sin \delta-\frac{w_{f}}{2} \cos \delta\right)-\frac{w_{r}}{2} F_{x r l}+ \\
F_{x f r}\left(l_{f} \sin \delta+\frac{w_{f}}{2} \cos \delta\right)+\frac{w_{r}}{2} F_{x r r} \\
M_{F y}=l_{f} F_{y f} \cos \delta-l_{r} F_{y r}
\end{gathered}
$$

式中, $l_{f}$ 与 $l_{r}$ 分别表示前轴与后轴到车辆质心的距 离, $w_{i}$ 表示轮距, $F_{x i j}=T_{q i j} / R_{e}$ 为第 $i j$ 个车轮上的纵 向力。

轮胎的侧向力主要由有效侧偏刚度与侧偏角决 定, 因而侧向力的导数

$$
\dot{F}_{y j}=K_{y c, i j} \dot{j}_{i j}+\dot{K}_{y c, i j} \alpha_{i j}
$$

轮胎侧偏角可表示为

$$
\alpha_{i j}=\arctan \left(\beta+\frac{\zeta_{i} l_{i} r}{u}\right)-\delta_{i}
$$

式中, $\zeta_{f}=-\zeta_{r}=1, \delta_{f}=\delta, \delta_{r}=0$ 。

对轮胎侧偏角求导可得 ${ }^{[19]}$

$$
\begin{gathered}
\dot{\alpha}_{i j} \approx \hat{A}_{i j} \dot{r} \\
\hat{A}_{i j}=\frac{\zeta_{i} l_{i} / u}{1+\left(\beta+\frac{\zeta_{i} l_{i} r}{u}\right)^{2}}
\end{gathered}
$$

$K_{y c}$ 与 $\dot{K}_{y c}$ 在第 1.2 节中给出, $\dot{K}_{y c}$ 可重新写为

$$
\dot{K}_{y c}=\tilde{K}_{y} \frac{\mathrm{d} S_{x}}{\mathrm{~d} \kappa} \frac{\mathrm{d} \kappa}{\mathrm{d} t}=\tilde{K}_{y} \frac{1}{(1+\kappa)^{2}} \dot{\kappa}
$$




$$
\dot{\kappa}_{i j}=-\frac{1}{\omega_{i j}^{*}} \dot{e}_{\omega i j}+\frac{e_{\omega i j}}{\omega_{i j}^{* 2}} \dot{\omega}_{i j}^{*}
$$

式中, $e_{\omega i j}$ 及其导数可表示为

$$
\left\{\begin{array}{l}
e_{\omega i j}=\omega_{i j}^{*}-\omega_{i j} \\
\dot{e}_{\omega i j}=\dot{\omega}_{i j}^{*}-\dot{\omega}_{i j}
\end{array}\right.
$$

式中, $\omega_{i j}$ 表示车轮实际滚动角速度, 由传感器测量 获得, 车轮角加速度可由车轮动力学计算

$$
\dot{\omega}_{i j}=\frac{1}{I_{w}}\left(T_{q i j}-\hat{F}_{x i j} R_{e}\right)
$$

式中, $\hat{F}_{x i j}$ 为估计的轮胎纵向力, 可由轮胎力估计算 法得到 ${ }^{[26]}, I_{w}$ 是车轮转动惯量。 $\omega_{i j}^{*}$ 表示期望的车轮 滚动角速度, 由轮心速度计算得到

$$
\omega_{i j}^{*}=\frac{u}{R_{e}}+\frac{r \varsigma_{j} w_{i}}{2 R_{e}}
$$

其导数

$$
\dot{\omega}_{i j}^{*}=\frac{a_{x}}{R_{e}}+\frac{\dot{r} \varsigma_{j} w_{i}}{2 R_{e}}
$$

式中, $\varsigma_{r}=-\varsigma_{l}=1, a_{x}$ 是纵向加速度。

然后由式(27)、(28)可得式(23)的第二项为

$$
\begin{gathered}
\dot{K}_{y c, i j} \alpha_{i j}=k_{i j} e_{\omega i j}+\tilde{k}_{i j} \dot{e}_{\omega i j} \\
k_{i j}=\frac{\tilde{K}_{y, i j} \dot{\omega}_{i j}^{*} \alpha_{i j}}{\left(1+\kappa_{i j}\right)^{2} \omega_{i j}^{* 2}} \\
\tilde{k}_{i j}=-\frac{\tilde{K}_{y, i j} \alpha_{i j}}{\left(1+\kappa_{i j}\right)^{2} \omega_{i j}^{*}}
\end{gathered}
$$

根据上述结果可得预测模型的状态空间形式为

$$
\boldsymbol{G}_{c} \dot{\boldsymbol{X}}=\boldsymbol{A}_{c} \boldsymbol{X}+\boldsymbol{B}_{c} \boldsymbol{U}+\boldsymbol{D}_{c} \boldsymbol{W}
$$

式中, $W$ 为干扰矢量。

$$
\boldsymbol{W}=\left(\begin{array}{lllll}
a_{x} & \tilde{F}_{x f l} & \tilde{F}_{x f r} & \tilde{F}_{x r l} & \tilde{F}_{x r r}
\end{array}\right)^{\mathrm{T}}
$$

矩阵 $\boldsymbol{A}_{c} 、 \boldsymbol{B}_{c} 、 \boldsymbol{D}_{c} 、 \boldsymbol{G}_{c}$ 定义如下

$$
\boldsymbol{A}_{c(8 \times 8)}=\left(\begin{array}{cccccccc}
-a_{x} m-u m & \cos \delta & 1 & 0 & 0 & 0 & 0 \\
0 & 0 & l_{f} \cos \delta & -l_{r} & 0 & 0 & 0 & 0 \\
0 & 0 & 0 & 0 & k_{f l} & k_{f r} & 0 & 0 \\
0 & 0 & 0 & 0 & 0 & 0 & k_{r l} & k_{r r} \\
0 & 0 & 0 & 0 & 0 & 0 & 0 & 0 \\
0 & 0 & 0 & 0 & 0 & 0 & 0 & 0 \\
0 & 0 & 0 & 0 & 0 & 0 & 0 & 0 \\
0 & 0 & 0 & 0 & 0 & 0 & 0 & 0
\end{array}\right)
$$

$$
\boldsymbol{B}_{c(8 \times 4)}=\left(\begin{array}{cccc}
\frac{\sin \delta}{R_{e}} & \frac{\sin \delta}{R_{e}} & 0 & 0 \\
B_{1} & B_{2} & \frac{-w_{r}}{2 R_{e}} & \frac{w_{r}}{2 R_{e}} \\
0 & 0 & 0 & 0 \\
0 & 0 & 0 & 0 \\
-\frac{1}{I_{w}} & 0 & 0 & 0 \\
0 & -\frac{1}{I_{w}} & 0 & 0 \\
0 & 0 & -\frac{1}{I_{w}} & 0 \\
0 & 0 & 0 & -\frac{1}{I_{w}}
\end{array}\right)
$$

$$
B_{1}=\frac{l_{f} \sin \delta-\frac{w_{f}}{2} \cos \delta}{R_{e}}
$$

$$
B_{2}=\frac{l_{f} \sin \delta+\frac{w_{f}}{2} \cos \delta}{R_{e}}
$$

$$
\boldsymbol{D}_{c(8 \times 5)}=\left(\begin{array}{ccccc}
0 & 0 & 0 & 0 & 0 \\
0 & 0 & 0 & 0 & 0 \\
0 & 0 & 0 & 0 & 0 \\
0 & 0 & 0 & 0 & 0 \\
\frac{1}{R_{e}} & \frac{R_{e}}{I_{w}} & 0 & 0 & 0 \\
\frac{1}{R_{e}} & 0 & \frac{R_{e}}{I_{w}} & 0 & 0 \\
\frac{1}{R_{e}} & 0 & 0 & \frac{R_{e}}{I_{w}} & 0 \\
\frac{1}{R_{e}} & 0 & 0 & 0 & \frac{R_{e}}{I_{w}}
\end{array}\right)
$$

$$
\boldsymbol{G}_{c(8 \times 8)}=\left(\begin{array}{cccccccc}
u m & 0 & 0 & 0 & 0 & 0 & 0 & 0 \\
0 & I_{\mathrm{z}} & 0 & 0 & 0 & 0 & 0 & 0 \\
0 & G_{1} & 1 & 0 & -\tilde{k}_{f l} & -\tilde{k}_{f r} & 0 & 0 \\
0 & G_{2} & 0 & 1 & 0 & 0 & -\tilde{k}_{r l}-\tilde{k}_{r r} \\
0 & \frac{w_{f}}{2 R_{e}} & 0 & 0 & 1 & 0 & 0 & 0 \\
0 & -\frac{w_{f}}{2 R_{e}} & 0 & 0 & 0 & 1 & 0 & 0 \\
0 & \frac{w_{r}}{2 R_{e}} & 0 & 0 & 0 & 0 & 1 & 0 \\
0-\frac{w_{r}}{2 R_{e}} & 0 & 0 & 0 & 0 & 0 & 1
\end{array}\right)
$$




$$
\begin{aligned}
& G_{1}=-\left(K_{y c, f l} \hat{A}_{f l}+K_{y c, f r} \hat{A}_{f r}\right) \\
& G_{2}=-\left(K_{y c, r l} \hat{A}_{r l}+K_{y c, r r} \hat{A}_{r r}\right)
\end{aligned}
$$

\section{2 车辆期望状态}

在跟踪控制中通常将线性二自由度车辆模型作 为理想模型 ${ }^{[27]}$, 本文将线性二自由度模型得到的质 心侧偏角和横摆角速度作为期望状态用于控制器设 计。单轨二自由度车辆模型运动方程为

$$
\begin{gathered}
\dot{\beta}=\frac{1}{m u}\left(F_{y f}+F_{y r}\right)-r \\
\dot{r}=\frac{1}{I_{z}}\left(l_{f} F_{y f}-l_{r} F_{y r}\right)
\end{gathered}
$$

由于 $\delta$ 值较小, 此处假设 $\sin \delta=0, \cos \delta=1$ 。 由式(24)可得前后轮侧偏角为

$$
\begin{gathered}
\alpha_{f} \approx \beta+\frac{l_{f} r}{V_{x}}-\delta \\
\alpha_{r} \approx \beta-\frac{l_{r} r}{V_{x}}
\end{gathered}
$$

然后，构造线性二自由度模型的状态空间形式

$$
\left(\begin{array}{l}
\dot{\beta} \\
\dot{r}
\end{array}\right)=\left(\begin{array}{ll}
a_{11} & a_{12} \\
a_{21} & a_{22}
\end{array}\right)\left(\begin{array}{l}
\beta \\
r
\end{array}\right)+\left(\begin{array}{l}
d_{1} \\
d_{2}
\end{array}\right) \delta
$$

式中, $a_{11}=-2 \frac{K_{y f}+K_{y r}}{m u}, a_{12}=2 \frac{l_{r} K_{\mathrm{yr}}-l_{f} K_{y f}}{m u^{2}}-1$, $a_{21}=2 \frac{l_{r} K_{y r}-l_{f} K_{y f}}{I_{z}} \quad, \quad a_{22}=-2 \frac{l_{f}^{2} K_{y f}+l_{r}^{2} K_{y r}}{I_{z} u}$, $d_{1}=\frac{2 K_{y f}}{m u}, d_{2}=\frac{2 l_{f} K_{y f}}{I_{z}} 。 K_{y f} 、 K_{y r}$ 分别为前轮和后 轮的侧偏刚度。

通过对式(50)进行拉普拉斯变换可得

$$
\begin{aligned}
& \beta(s)=G_{B} \frac{1+T_{B} s}{1-\left(T_{A} / D_{A}\right) s+\left(1 / D_{A}\right) s^{2}} \delta(s) \\
& r(s)=G_{R} \frac{1+T_{R} s}{1-\left(T_{A} / D_{A}\right) s+\left(1 / D_{A}\right) s^{2}} \delta(s)
\end{aligned}
$$

式中, $G_{B}=\frac{d_{2} a_{12}-d_{1} a_{22}}{D_{A}}, T_{B}=\frac{d_{1}}{d_{2} a_{12}-d_{1} a_{22}}$, $G_{R}=\frac{d_{1} a_{21}-d_{2} a_{11}}{D_{A}}, \quad T_{R}=\frac{d_{2}}{d_{1} a_{21}-d_{2} a_{11}}, \quad T_{A}=a_{11}+a_{22}$, $D_{A}=a_{11} a_{22}-a_{12} a_{21}$ 。

由式(51)、(52)可得稳态值

$$
\begin{aligned}
& \beta_{s s}=G_{B} \delta \\
& r_{s s}=G_{R} \delta
\end{aligned}
$$

考虑由于路面附着极限导致的轮胎力饱和, 最 大稳态横摆角速度为

$$
r_{\max }=\frac{\mu g}{V_{x}}
$$

然后，考虑路面附着约束的稳态横摆角速度和 质心侧偏角可描述为

$$
\begin{gathered}
r_{s s c}= \begin{cases}G_{R} \delta & G_{R} \delta \mid \leqslant r_{\max } \\
r_{\max } \operatorname{sgn}\left(G_{R} \delta\right) & \text { 其他 }\end{cases} \\
\beta_{s s c}=\frac{G_{B}}{G_{R}} r_{s s c}
\end{gathered}
$$

最终，将式(56)、(57)代入式(51)、(52)中可以 得到考虑路面附着约束的期望车辆状态

$$
\begin{aligned}
& \beta_{d}(s)=\frac{1+T_{B} s}{1-\left(T_{A} / D_{A}\right) s+\left(1 / D_{A}\right) s^{2}} \beta_{s s c}(s) \\
& r_{d}(s)=\frac{1+T_{R} s}{1-\left(T_{A} / D_{A}\right) s+\left(1 / D_{A}\right) s^{2}} r_{s s c}(s)
\end{aligned}
$$

\section{3 车辆稳定性指标}

相平面作为一种图形化的方法能够通过车辆状 态在相平面中所处的位置来判断车辆的稳定性。本 文采用轮胎侧偏角 $\left(\alpha_{f}-\alpha_{r}\right)$ 相平面对车辆的稳定性进 行分析, 根据第 2.2 节中给出的二自由度车辆运动 方程和第 1.1 节中介绍的 UniTire 轮胎模型, 可得图 9、10 所示的相平面。图 9、10 中对角深色阴影部 分表示不稳定区域, 圆表示鞍点位置, 垂直虚线表 示前轮最大侧向力对应的侧偏角 $\alpha_{f, s a t}$, 水平虚线表 示后轮最大侧向力对应的侧偏角 $\alpha_{r}$, sat

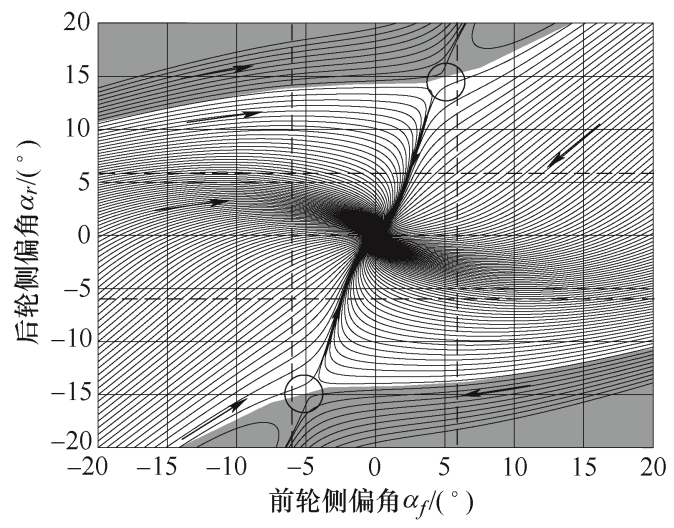

图 9 轮胎侧偏角相平面: $u=15 \mathrm{~m} / \mathrm{s}, \mu=0.8, \delta=0^{\circ}$

可以看到图 10 中的鞍点位置相对于图 9 发生了 移动, 这是因为图 10 的前轮转角发生了变化。实际 上相平面中的鞍点位置受到纵向速度 $u$, 路面摩擦 因数 $\mu$ 和前轮转角 $\delta$ 共同作用的影响 ${ }^{[9]}$ 。文献[28] 给出了 $\beta-r$ 相平面中鞍点 $S_{1}\left(\beta_{s 1}, r_{s 1}\right)$ 和 $S_{2}\left(\beta_{s 2}, r_{s 2}\right)$ 的 


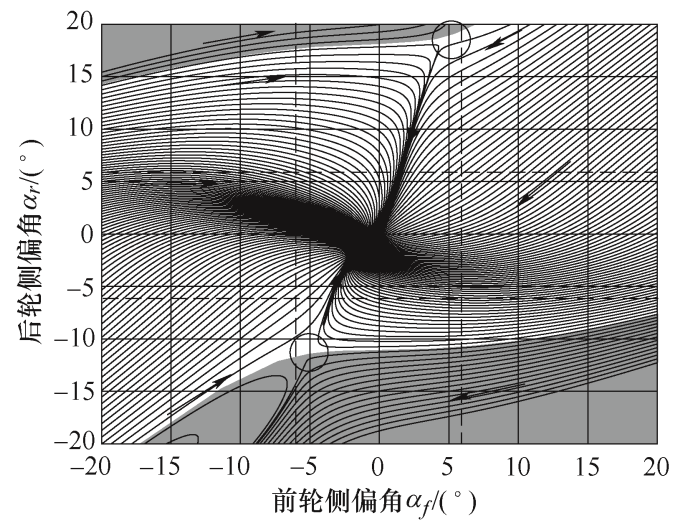

图 10 轮胎侧偏角相平面: $u=15 \mathrm{~m} / \mathrm{s}, \mu=0.8, \delta=4.5^{\circ}$

确定方法, 如式(60) (63)所示

$$
\begin{gathered}
r_{s 1}=\frac{\mu g}{V_{x}} \\
\beta_{s 1}=-\frac{l_{f} r}{V_{x}}+\tan \left(-\alpha_{f, s a t}+\delta\right) \\
r_{s 2}=-\frac{\mu g}{V_{x}} \\
\beta_{s 2}=-\frac{l_{f} r}{V_{x}}+\tan \left(\alpha_{f, s a t}+\delta\right)
\end{gathered}
$$

式中, $\alpha_{f, s a t}$ 和 $\alpha_{r, s a t}$ 分别为前轮和后轮最大侧向力对 应的侧偏角。

$\alpha_{f}-\alpha_{r}$ 相平面中的鞍点位置可以由式(48)、 通过简单的代数转换得到。

在 $\alpha_{f}-\alpha_{r}$ 相平面中, $X$ 轴与 $Y$ 轴方向都呈现的是 轮胎侧偏角状态, 因而其中的稳定性区域分布比较 均匀, 更加适合车辆的稳定性分析。因此车辆的稳 定程度可以通过车辆当前状态在相平面中所处的位 置进行评估。基于此, 本文提出了一种定量的车辆 稳定性评价指标(图 11)。最小半径圆形区域表示稳 定区域, 它是以原点为中心 $R_{1}=\alpha_{f, s a t}$ 为半径的圆。 当状态位于该区域时车辆是稳定的，一般情况下前 轮饱和侧偏角要大于后轮饱和侧偏角, 因而该区域 允许后轮侧偏有一定的超调量。

稳定区域边界到邻近的圆形边界之间的区域部 分表示过渡区域, 它是以原点为圆心, $R_{2}=\sqrt{\alpha_{f, s a t}^{2}+\alpha_{r, s a t}^{2}}$ 为半径的圆。从相平面的角度来看 该区域也是稳定的, 但是该区域中车辆的前后轮侧 偏角都达到了较大的值, 并且一部分超过饱和侧偏 角值, 因而车辆失稳的可能性增大。

最大半径圆形区域和过渡区域之间为不稳定区 域, 它是以原点为圆心, 半径为 $R_{3}=\sqrt{\alpha_{f, s}^{2}+\alpha_{r, s}^{2}}$ 的 圆, $\left(\alpha_{f, s}, \alpha_{r, s}\right)$ 表示靠近圆心的鞍点坐标。当状态位于
该区域时车辆被认为是失稳的, 此时前后轮侧偏角 都已经进入严重饱和区域。

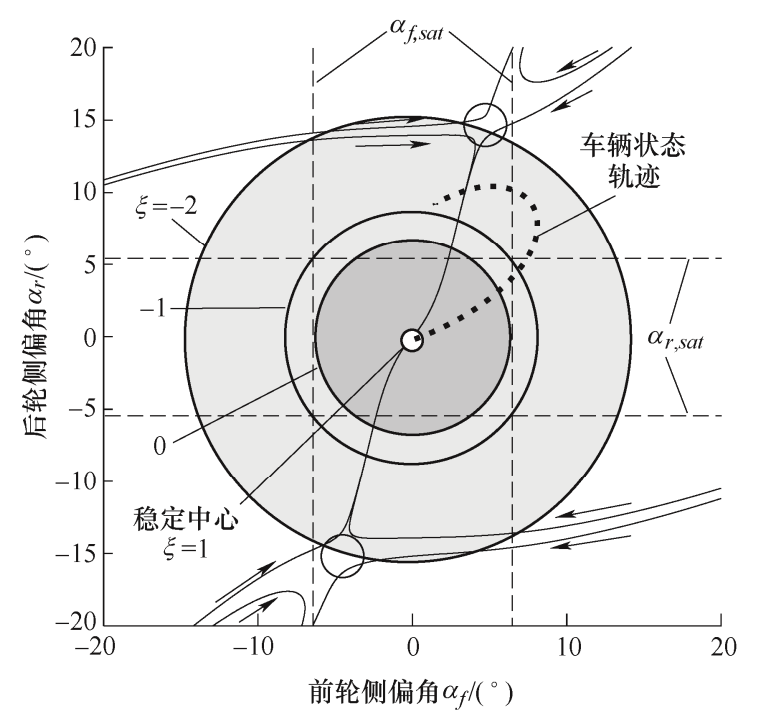

图 11 轮胎侧偏角相平面的不同区域划分

然后, 车辆稳定性指标

$$
\xi= \begin{cases}\frac{R_{1}-R_{\alpha}}{R_{1}} & R_{\alpha} \leqslant R_{1} \\ \frac{-R_{\alpha}}{R_{2}-R_{1}}+\frac{R_{1}}{R_{2}-R_{1}} & R_{1}<R_{\alpha} \leqslant R_{2} \\ \frac{-R_{\alpha}}{R_{3}-R_{2}}+\frac{R_{2}}{R_{3}-R_{2}}-1 & R_{2}<R_{\alpha} \leqslant R_{3}\end{cases}
$$

式中, $R_{\alpha}=\sqrt{\alpha_{f}^{2}+\alpha_{r}^{2}}$ 表示当前车辆状态到原点位置 的距离。可以看到当 $\xi \in(0,1]$ 时, 车辆状态是稳定 的, $\xi$ 数值越大, 稳定程度越高。

当 $\xi \in(-1,0]$ 时, 车辆状态位于过渡区域, 表示 轮胎侧偏角即将或者是已经超过饱和值, 车辆失稳 的可能性增大。当 $\xi \in[-2,-1]$ 时, 车辆失稳, 前后 轮侧偏角都位于饱和区域, 转向盘转角已经不能控 制车辆的侧向运动。因此, 稳定性指标 $\xi$ 能够区分 车辆的稳定程度, 给出定量的车辆的稳定值。

\subsection{MPC 控制器}

本文采用 MPC 控制器实现多目标控制。首先 通过跟踪期望的车辆状态 $r_{d}$ 与 $\beta_{d}$ 用以提升车辆的操 纵性和稳定性, 然后对轮胎的滑移率进行约束防止 轮胎与地面出现滑转。这些控制目标构成 MPC 优 化问题的目标方程。

根据连续系统状态方程式(36)，可得离散化的 系统状态空间方程为

$$
\left\{\begin{array}{l}
\boldsymbol{X}^{k+1}=\boldsymbol{A}_{d} \boldsymbol{X}^{k}+\boldsymbol{B}_{d} \boldsymbol{U}^{k}+\boldsymbol{D}_{d} \boldsymbol{W}^{k} \\
\boldsymbol{Y}^{k}=\boldsymbol{C}_{d} \boldsymbol{X}^{k}
\end{array}\right.
$$

式中, $k$ 为离散步, 此处假设在预测时域内, 前轮 
转角 $\delta$ 、纵向速度 $u$ 是不变的。 $\boldsymbol{A}_{d} \in \mathbf{R}_{8 \times 8}$, $\boldsymbol{B}_{d} \in \mathbf{R}_{8 \times 4}, \boldsymbol{D}_{d} \in \mathbf{R}_{8 \times 5}$ 表示系统矩阵。

$$
\begin{gathered}
\boldsymbol{A}_{d}=\boldsymbol{I}+\boldsymbol{A}_{c} \Delta T \\
\boldsymbol{B}_{d}=\boldsymbol{B}_{c} \Delta T \\
\boldsymbol{D}_{d}=\boldsymbol{D}_{c} \Delta T
\end{gathered}
$$

式中, $\Delta T$ 为离散时间步长, $I$ 为单位矩阵。 $\boldsymbol{Y}$ 为系 统输出矢量, 表示需要被约束的车辆状态, $\boldsymbol{C}_{d}$ 输出 矩阵, 分别为

$$
\begin{aligned}
\boldsymbol{Y} & =\left(\begin{array}{lllllll}
\beta & r & e_{\omega f l} & e_{\omega f r} & e_{\omega r l} & e_{\omega r r}
\end{array}\right)^{\mathrm{T}} \\
\boldsymbol{C}_{d} & =\left(\begin{array}{llllllllll}
1 & 0 & 0 & 0 & 0 & 0 & 0 & 0 \\
0 & 1 & 0 & 0 & 0 & 0 & 0 & 0 \\
0 & 0 & 0 & 0 & 1 & 0 & 0 & 0 \\
0 & 0 & 0 & 0 & 0 & 1 & 0 & 0 \\
0 & 0 & 0 & 0 & 0 & 0 & 1 & 0 \\
0 & 0 & 0 & 0 & 0 & 0 & 0 & 1
\end{array}\right)
\end{aligned}
$$

MPC 优化问题可以被构造为

$$
\left\{\begin{array}{l}
J=\frac{1}{2}\left\|\boldsymbol{Y}^{N p}-\boldsymbol{Y}_{d}^{N p}\right\|_{\boldsymbol{Q}}^{2}+ \\
\frac{1}{2} \sum_{k=0}^{N p-1}\left(\left\|\boldsymbol{Y}^{k}-\boldsymbol{Y}_{d}^{k}\right\|_{\boldsymbol{Q}}^{2}+\left\|\boldsymbol{U}^{k}-\boldsymbol{U}^{r e f}\right\|_{\boldsymbol{R}}^{2}+\left\|\boldsymbol{U}^{k}-\boldsymbol{U}^{k-1}\right\|_{\boldsymbol{T}}^{2}\right) \\
\overline{\boldsymbol{U}}^{*}=\arg \min (J)
\end{array}\right.
$$

式中, $\|\boldsymbol{s}\|_{\boldsymbol{Q}}^{2}=\boldsymbol{s}^{\mathrm{T}} \boldsymbol{Q} \boldsymbol{s}$ 表示加权 2 范数, $N_{p}$ 表示预测 步长, $\boldsymbol{Y}_{d}=\left(\begin{array}{llllll}\beta_{d} & r_{d} & 0 & 0 & 0 & 0\end{array}\right)^{\mathrm{T}}$ 表示期望状态, $\boldsymbol{U}^{k}$ 表 示第 $k$ 步的预测输出, $\boldsymbol{U}^{r e f}$ 表示参考的四轮力矩平 均分配, 由驾驶员总驱动力矩需求计算得到, 确 保车辆在直线行驶等常规工况下车辆性能的发 挥, 并且能够提高优化求解收玫速度; $\boldsymbol{U}^{k-1}$ 表示 前一时刻控制器输出结果, 减小预测控制幅度, 用于对输出结果的变化率进行限制; $\bar{U}^{*}$ 为预测输 出结果。正定矩阵 $\boldsymbol{Q} 、 \boldsymbol{R} 、 \boldsymbol{T}$ 为权重矩阵, 根据控 制需求进行合理设置, 反映各个控制目标的重要 性, 可表示为

$$
\begin{aligned}
& \boldsymbol{Q}=\operatorname{diag}\left(q_{1} \quad q_{2} q_{3} \quad q_{4} q_{5} q_{6}\right) \\
& \boldsymbol{R}=\operatorname{diag}\left(\begin{array}{llll}
q_{u} & q_{u} & q_{u} & q_{u}
\end{array}\right) \\
& \boldsymbol{T}=\operatorname{diag}\left(q_{p} \quad q_{p} \quad q_{p} \quad q_{p}\right)
\end{aligned}
$$

然后采用文献[29]介绍的方法进行数值求解, 目标方程可重写为

$$
J(\overline{\boldsymbol{U}})=\frac{1}{2} \overline{\boldsymbol{U}}^{\mathrm{T}} \boldsymbol{H} \overline{\boldsymbol{U}}+\overline{\boldsymbol{U}}^{\mathrm{T}} \boldsymbol{g}\left(\boldsymbol{X}_{0}\right)+c
$$

式中, $\boldsymbol{H}$ 由预测模型和权重矩阵决定是一个对称矩 阵, 矢量 $\boldsymbol{g}\left(\boldsymbol{X}_{0}\right)$ 是由系统初始状态, 系统矩阵以及权 重矩阵决定的矢量, $c$ 为常数。 $\bar{U}$ 为预测时域内的 控制序列, 求解结果为

$$
\overline{\boldsymbol{U}}^{\mathrm{T}}=\left(\begin{array}{llll}
\overline{\boldsymbol{U}}_{0}^{\mathrm{T}} & \overline{\boldsymbol{U}}_{1}^{\mathrm{T}} & \cdots & \overline{\boldsymbol{U}}_{N p}^{\mathrm{T}}
\end{array}\right)
$$

最终控制器实际输出结果为 $\overline{\boldsymbol{U}}_{0}^{\mathrm{T}}$ 。

\section{5 控制器约束}

MPC 控制结构的优点在于能够实现考虑约束 情况下的多目标控制, 为了满足不同工况下车辆的 控制需求，本节对系统约束进行设计。

\subsection{1 操纵性和稳定性约束}

从控制的角度可知跟踪两个系统状态(期望的 质心侧偏角 $\beta_{d}$ 和横摆角速度 $r_{d}$ 需要两个独立的控 制输入 ${ }^{[30]}$, 四轮驱动电动汽车虽然四个车轮力矩独 立可调但是产生的控制输入都是横摆力矩 $M_{z}$, 此时 实际上是对两个状态跟踪的一种折中, 即根据权重 来调节两个状态的跟踪程度。通常横摆角速度侧重 表征车辆的操纵性, 而质心侧偏角侧重表征车辆的 稳定性。当车辆状态位于稳定区域时, 通常期望车 辆具有较好的操纵性, 此时以操纵性控制为主要目 标; 当车辆状态位于不稳定区域时, 此时要优先考 虑稳定性控制目标。因此质心侧偏角跟踪权重 $q_{1}$ 和 横摆角速度跟踪权重 $q_{2}$ 被设计成稳定性指标 $\xi$ 的函 数, 分别为

$$
\begin{aligned}
& q_{1}= \begin{cases}s_{1} \xi^{2}+s_{2} & \xi \leqslant 0 \\
s_{2} & \xi>0\end{cases} \\
& q_{2}= \begin{cases}s_{3}-s_{4} q_{1} & \xi \leqslant 0 \\
s_{3}-s_{2} & \xi>0\end{cases}
\end{aligned}
$$

式中, $s_{1} \sim s_{4}$ 为参数, 通过调试分别取值为 $s_{1}=400$, $s_{2}=1, s_{3}=400, s_{4}=0.85$ 控制效果较好。变化效果见 图 12。

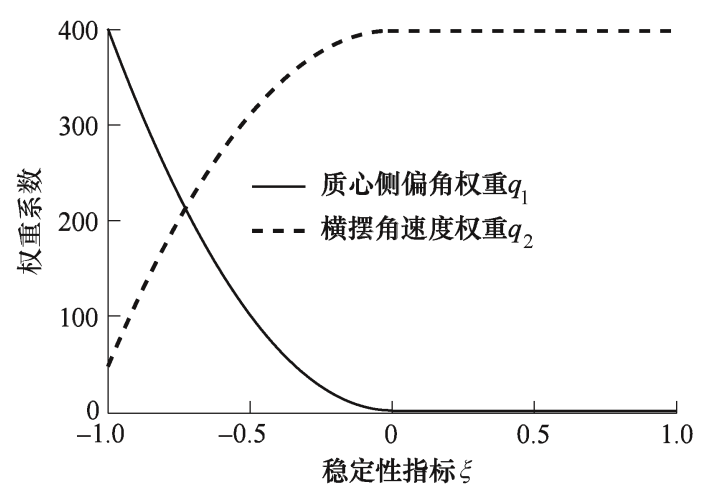

图 12 质心侧偏角和横摆角速度权重随 $\xi$ 的变化 
由第 2.3 节的定义可知, 当 $\xi>0$ 时车辆状态处 于稳定区域, $q_{1}$ 取值较小, $q_{2}$ 取值较大, 此时以操 纵性为控制目标; 当 $\xi \leqslant 0$ 时车辆状态处于过渡或失 稳区域, $q_{1}$ 取值增大, $q_{2}$ 取值减小, 从而实现稳定 性控制。

\subsection{2 轮胎滑移率约束}

为了防止在驱动和制动时轮胎出现打滑的情 况, 对车轮滑移率进行约束。将约束权重 $q_{n}(n=3,4$, $5,6)$ 作为滑移率 $\kappa$ 的函数, 如式(79)所示

$$
q_{n}=\left[a_{h} \cdot\left(\frac{|\kappa|}{p_{2}}-p_{1}\right)+\sqrt{\left(a_{h} \cdot\left(\frac{|\kappa|}{p_{2}}-p_{1}\right)\right)^{2}+b_{h}^{2}}\right] / a_{h}+c_{h}
$$

式(79)为双曲线方程, 其中 $a_{h}=30 、 b_{h}=3 、 c_{h}=0.5$ 、 $p_{1}=0.5 、 p_{2}=0.2$ 为参数, $q_{n}$ 与 $\kappa$ 的关系见图 13 。当 滑移率小于 0.1 时, 权重值很小, 而滑移率超过 0.1 后, 权重值迅速增大。

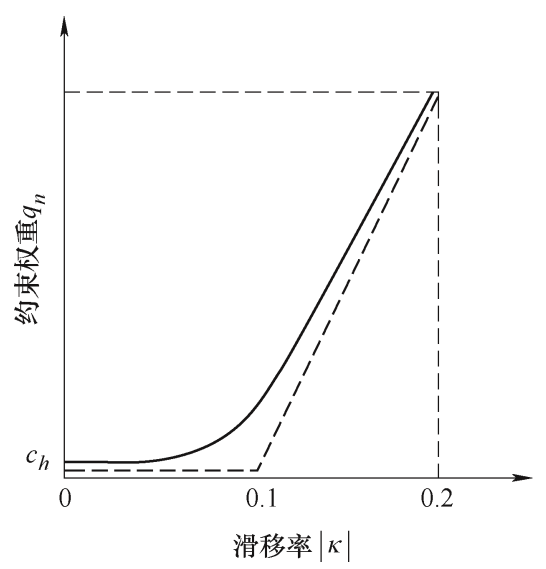

图 13 轮胎滑移率双曲线权重约束效果

\subsection{3 执行器约束}

驱动电机由于物理限制存在最大驱动力矩 $\boldsymbol{T}_{d}$ 以及最大制动力矩 $\boldsymbol{T}_{b}$ 。因而在预测时域内的控制量 $\bar{U}$ 受到如下约束。

$$
\begin{gathered}
\boldsymbol{L}_{b} \leqslant \overline{\boldsymbol{U}} \leqslant \boldsymbol{U}_{b} \\
\left\{\begin{array}{l}
\boldsymbol{L}_{b}^{\mathrm{T}}=\underbrace{\left(\boldsymbol{T}_{b}^{\mathrm{T}} \boldsymbol{T}_{b}^{\mathrm{T}} \cdots \boldsymbol{T}_{b}^{\mathrm{T}}\right.}_{N p+1}) \\
\boldsymbol{U}_{b}^{\mathrm{T}}=\underbrace{\left(\boldsymbol{T}_{d}^{\mathrm{T}} \boldsymbol{T}_{d}^{\mathrm{T}} \cdots \boldsymbol{T}_{d}^{\mathrm{T}}\right.}_{N p+1})
\end{array}\right.
\end{gathered}
$$

式中, $\boldsymbol{L}_{b}$ 为约束下边界, $\boldsymbol{U}_{b}$ 为约束上边界。

如此控制器能够求解出符合执行器性能的输出 结果。

\subsection{4 不同约束关系分析}

操纵性约束与稳定性约束存在一定的制约关 系, 都是稳定性指标 $\xi$ 的函数, 通过稳定性指标来 调节。操纵性和稳定性是整车层面的性能, 除此之 外, 需要对轮胎的滑移率进行约束, 防止车轮过 度滑移, 滑移率约束是通过降低轮胎的力矩值实现 的, 当滑移率约束权重较大时会抑制轮胎力矩值增 大, 这样可能会对操纵性和稳定性控制造成负面影 响, 为了解决该问题, 将滑移率约束设置为双曲函 数的形式, 当滑移率较小时, 其权重值很小, 不对 操纵性和稳定性目标造成影响, 只有当滑移率超过 0.1 之后其权重值才增大, 对车轮力矩值进行约束。 执行器约束属于硬约束。

\section{3 仿真分析}

本节采用 Simulink-CarSim 联合仿真的方法对 控制算法的效果进行验证，选取 CarSim 中的 E-Class 车辆模型作为仿真模型, 车辆参数见表 1, 同时用第 1.1 节中介绍的 UniTire 轮胎模型替换 CarSim 自带的轮胎模型用于仿真。

\section{表 1 车辆仿真参数}

\begin{tabular}{lc}
\multicolumn{1}{c}{ 参数 } & 数值 \\
\hline 簧载质量 $M_{\mathrm{s}} / \mathrm{kg}$ & 1800 \\
非簧载质量 $M_{\mathrm{u}} / \mathrm{kg}$ & 140 \\
前轴距质心距离 $l_{\mathrm{f}} / \mathrm{m}$ & 1.016 \\
后轴距质心距离 $l_{\mathrm{r}} / \mathrm{m}$ & 1.524 \\
轮距 $w / \mathrm{m}$ & 1.5 \\
质心高度 $h / \mathrm{m}$ & 0.75 \\
绕 $x$ 轴的转动惯量 $I_{\mathrm{x}} /\left(\mathrm{kg} \cdot \mathrm{m}^{2}\right)$ & 800 \\
绕 $y$ 轴的转动惯量 $I_{\mathrm{y}} /\left(\mathrm{kg} \cdot \mathrm{m}^{2}\right)$ & 2300 \\
绕 $z$ 轴的转动惯量 $I_{z} /\left(\mathrm{kg} \cdot \mathrm{m}^{2}\right)$ & 2500 \\
车轮转动惯量 $I_{\mathrm{w}} /\left(\mathrm{kg} \cdot \mathrm{m}^{2}\right)$ & 1.1 \\
\hline
\end{tabular}

为了验证使用复合工况 UniTire 轮胎模型后的 控制效果，设置了两种控制模式：模式 A 采用常侧 偏刚度值, 不考虑轮胎复合特性(即不考虑纵向力对 侧偏刚度的影响); 模式 B 采用第 1.2 节推导的完整 的复合工况 UniTire 轮胎模型表达式。

\section{1 低附蛇形工况仿真}

仿真输入见图 $14 \sim 15$, 车辆在低附着 $(\mu=0.3)$ 路面上以初速度 $90 \mathrm{~km} / \mathrm{h}$ 行驶, 然后在 $1 \mathrm{~s}$ 到 $8 \mathrm{~s}$ 减 速到 $60 \mathrm{~km} / \mathrm{h}$, 速度调节采用的是 PI 调节器。同时 前轮转角以正弦变化。上述输入会使车辆进入侧偏、 纵滑复合工况。 


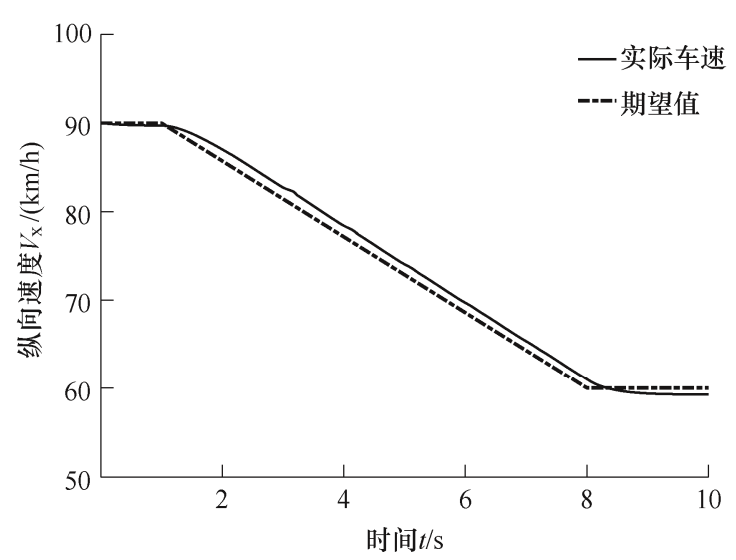

图 14 纵向速度

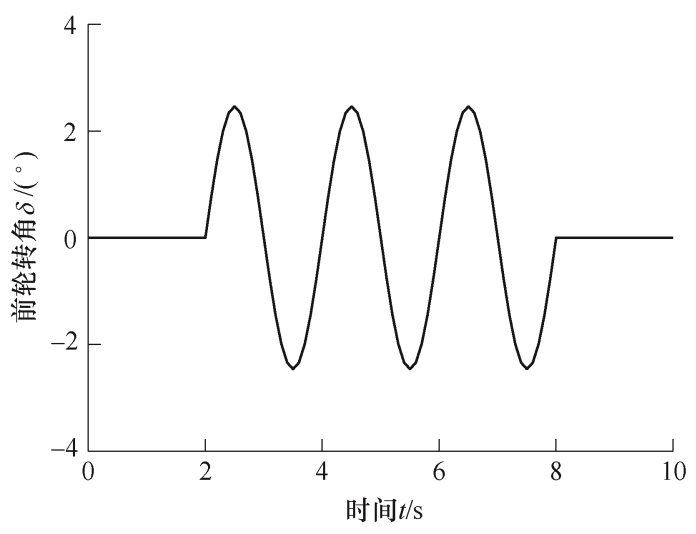

图 15 前轮转角

仿真结果见图 16 23, 图 16 显示了横摆角速 度控制效果, 可以看到不加控制时车辆失稳, 横摆 角速度 $r$ 无规律变化。控制器 $\mathrm{A}$ 和控制器 $\mathrm{B}$ 都很好 地跟踪上了参考值, 说明了 MPC 控制结构的有效 性。图 17 呈现了质心侧偏角变化曲线, 无控制时质 心侧偏角曲线发散, 控制器 $\mathrm{A}$ 和 $\mathrm{B}$ 的质心侧偏角被 约束在合理的范围内, 虽然没有严格跟踪上参考值, 但是其最大值(绝对值)为 $1.65^{\circ}$, 表明车辆具有很 好的稳定性。图 18 呈现了车辆稳定性指标 $\xi$ 随着车 辆状态的变化而发生相应的变化, 由于车辆处于强 动态, 因而车辆部分状态会进入临界稳定区域(即图 18 中 $\xi<0$ 的区域)。图 19 呈现的是质心侧偏角和横 摆角速度的跟踪权重, 由于这两个权重系数是 $\xi$ 的 函数, 因而随着 $\xi$ 的变化而自动调整, 曲线显示出 这两个权重具有自适应调节能力。同时可以看到由 于 $\xi$ 大部分位于稳定区域, 只有少部分进入到过渡 区域, 因而 $r$ 的跟踪权重取较大值, $\beta$ 的跟踪权重 取较小值, 控制器呈现较好的 $r_{d}$ 跟踪效果(图 16)。

图 20 展现的是控制器 $\mathrm{A}$ 的四轮滑移率, 图 21 显示 的是控制器 $B$ 的四轮滑移率, 可以看到控制器 $A$ 每 个周期的滑移率极值(绝对值)都明显大于控制器 $\mathrm{B}$, 控制器 $\mathrm{A}$ 的最大滑移率为 0.13 , 而控制器 $\mathrm{B}$ 的最大
值为 0.11 , 对比可以发现后轴两个车轮的滑移率差 别更明显。这是因为两种控制器决策出的四轮力矩 值不同, 分别见图 22 和图 23, 从这两个图中可以 看到, 控制器 $\mathrm{A}$ 决策出的同一侧(左侧或右侧)的力 矩值基本一致, 而控制器 $\mathrm{B}$ 决策出的后轴力矩明显 小于前轴力矩, 考虑到车辆在制动的情况下, 前轴 的垂向载荷增大附着能力增强, 后轴垂向载荷减小 附着能力减弱, 因而减少后轴的力矩分配有利于抑 制滑移率, 得到如图 21 所示的效果, 即同一侧轮胎 (同左侧或同右侧两个轮胎)的滑移率值更加接近。

图 20 与图 21 的对比结果证明了考虑轮胎复合特性 (即将复合工况 UniTire 轮胎模型用于 MPC 控制器设 计)时控制器 $\mathrm{B}$ 能够有效地将纵向力对侧向力的衰 减作用代入到决策过程中, 从而决策出更加合理的 四轮力矩值, 在保证相同的控制效果的条件下有效 地抑制四轮滑移率的增大。通过对比控制器 $\mathrm{A}$ 和 B 的四轮力矩值(图 22、23)可以发现采用控制器 $\mathrm{B}$ 的 另外一个优点是降低了整体的力矩需求, 以每个周 期的峰值点为评价指标(如图 23 所示, f1, f2, f3, f4 为第二个周期的四个峰值点), 分别对控制器 $\mathrm{A}$ (图 22) 和控制器 $\mathrm{B}$ (图 23)各自的所有峰值点进行绝对值累加 对比发现控制器 $\mathrm{B}$ 的累加值相对于控制器 $\mathrm{A}$ 要小 $13.7 \%$ 。这是因为车辆在转向时所需要的横摆力矩有两 个来源, 一个是由侧向力产生的 $M_{F y}$, 另一个是纵向 力产生的 $M_{F x}$, 由于复合工况下侧向力与纵向力的耦 合作用, 纵向力的增大会导致侧向力的衰减, 当转向 所需要的总横摆力矩不变时, 控制器 $\mathrm{A}$ 导致滑移率明 显增大(图 20), 侧向力衰减较多即 $M_{F y}$ 明显减小, 因 而需要更大的 $M_{F x}$ 来保证所需要的总横摆力矩。然而 控制器 B 有效地抑制了滑移率的增大(图 21), 从而使 侧向力衰减较少, 其能够提供的 $M_{F y}$ 比控制器 $\mathrm{A}$ 要大, 所以只需要较小的 $M_{F x}$ 即可满足总的横摆力矩需求。

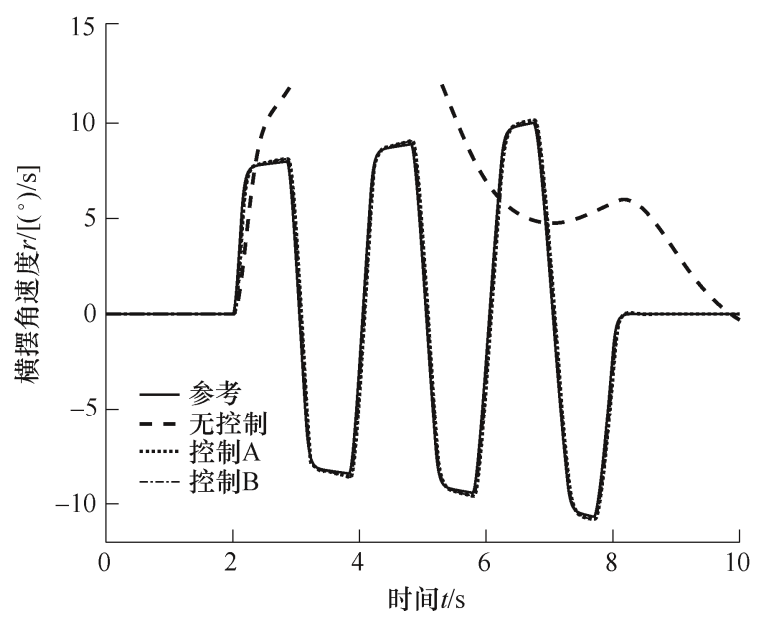

图 16 横摆角速度 


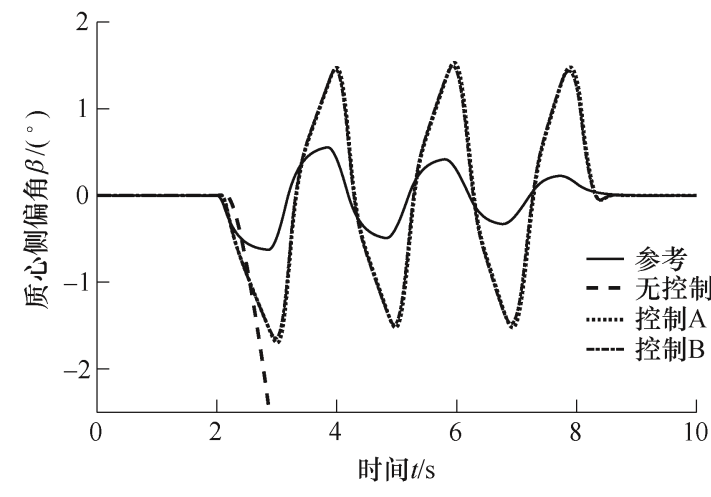

图 17 质心侧偏角

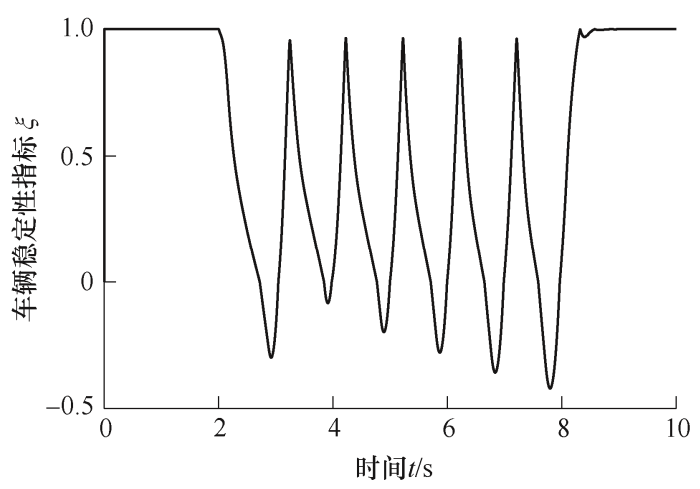

图 18 车辆稳定性指标 $\xi$

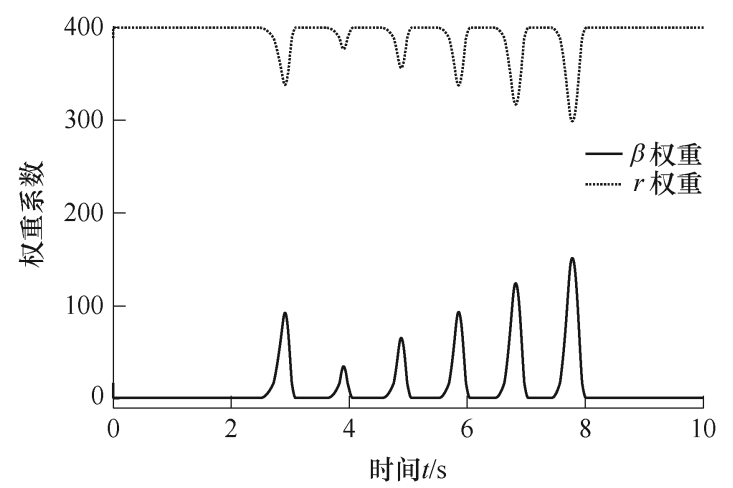

图 19 车辆质心侧偏角与横摆角速度跟踪权重系数

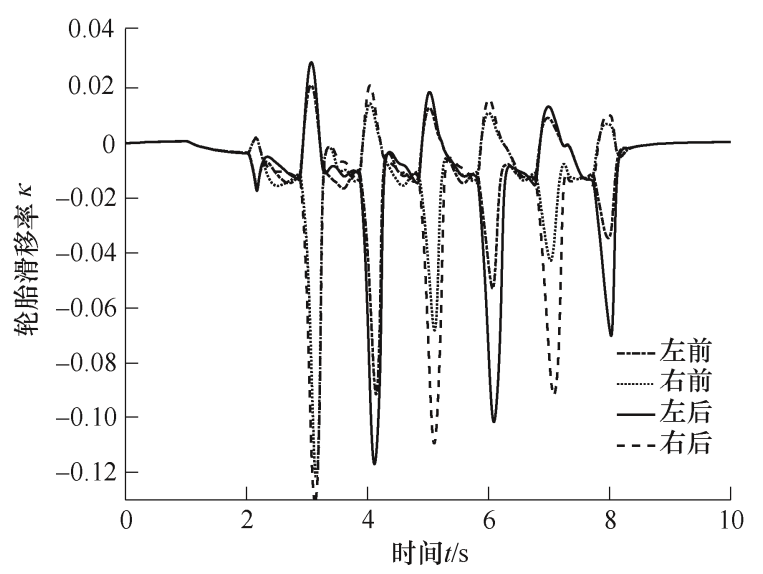

图 20 控制器 A：轮胎滑移率

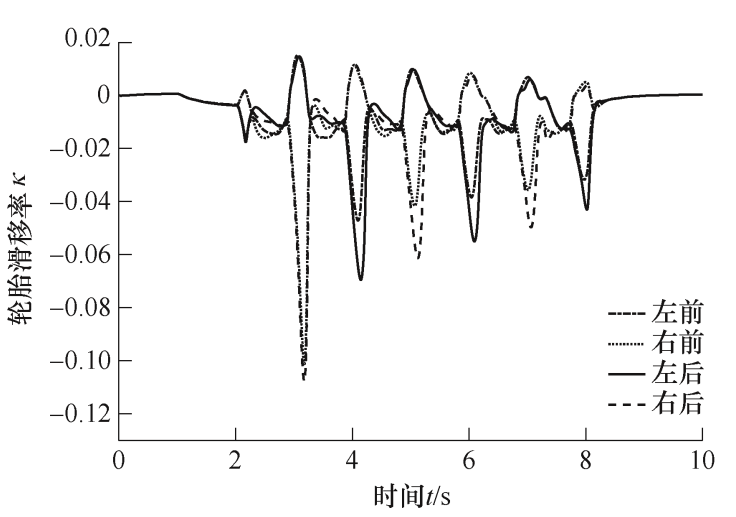

图 21 控制器 $\mathrm{B}$ : 轮胎滑移率

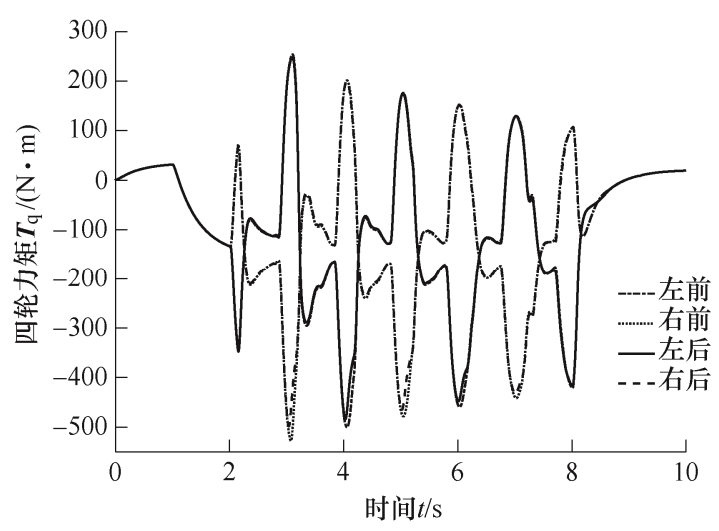

图 22 控制器 $\mathrm{A}$ : 四轮力矩分配

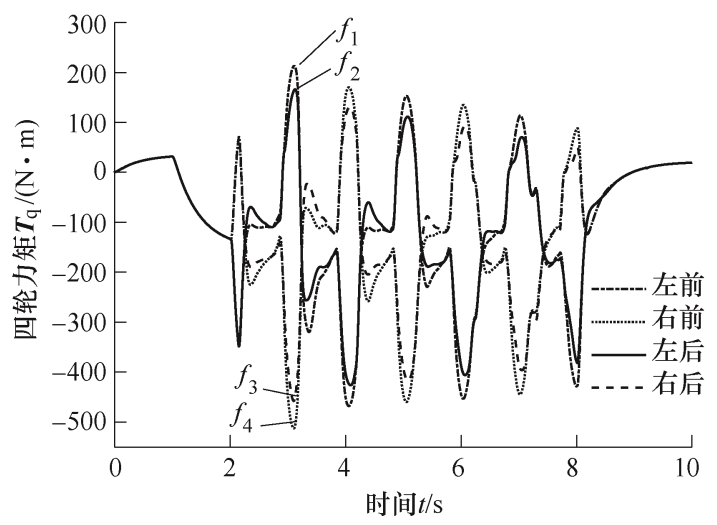

图 23 控制器 B: 四轮力矩分配

该仿真结果表明 MPC 控制器结构具有优异的 综合控制效果。通过合理的权重设置能够准确跟踪 上参考目标, 同时在控制器设计时采用复合轮胎模 型, 考虑纵向力对轮胎侧偏特性的影响, 能够使控 制器决策出更加合理的四轮力矩值, 减小四轮力矩 分配，抑制轮胎滑移率的增大。

\section{2 低附鱼钩输入工况仿真}

前轮转角输入见图 24, 前轮转角在前 $2 \mathrm{~s}$ 为向 右角阶跃输入，然后在 2 3 $\mathrm{s}$ 向左突然变化并保持 不变。速度变化与上一仿真工况相同见图 14。该输 入用于验证控制器 $\mathrm{B}$ 的极限工况控制效果。 


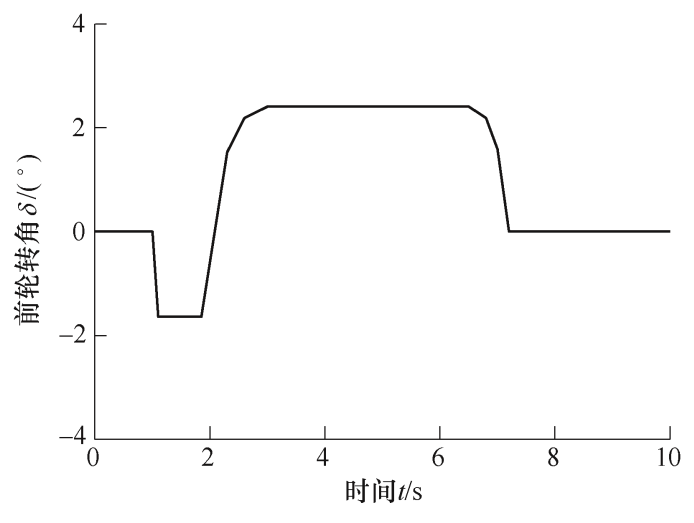

图 24 鱼钩前轮转角输入

仿真结果见图 25 30。图 25 为横摆角速度 变化曲线, 可以看到无控制时, 横摆角速度发散, 车辆失稳, 在控制器 $\mathrm{B}$ 作用下车辆在开始和结尾 阶段很好地跟踪上了参考值。图 26 为质心侧偏角 曲线, 无控制时质心侧偏角发散, 在控制器 $\mathrm{B}$ 的 作用下质心侧偏角最大值(绝对值)不超过 $2^{\circ}$, 表 明车辆是稳定的。图 27 为车辆的稳定性指标 $\xi$ 曲 线, 在 3 7 s 这段时间稳定性指标是负值, 并且 进入了 $\xi<-0.5$ 的区域, 表明车辆此时即将失稳。 图 28 为横摆角速度和质心侧偏角跟踪权重自适 应调节曲线, 由于在 3 7 s 这段时间稳定性指标 处于即将失稳的区域, 根据第 2.5 节的权重分配 结果, 质心侧偏角跟踪权重 $q_{1}$ 急剧增大, 横摆角 速度速度跟踪权重 $q_{2}$ 急剧减小。图 29 为轮胎滑 移率曲线, 由于控制器的多目标约束效果, 即使 在车辆即将失稳的情况下, 轮胎滑移率也被控制 在合理的范围内, 滑移率最大值约为 0.1 。图 30 为四轮力矩分配曲线, 后轴峰值力矩小于前轴, 有利于减小后轴轮胎滑移率。综合上述结果可以 发现该 MPC 控制器通过自适应调节 $\beta$ 与 $r$ 的跟踪 权重(图 28), 在车辆将要失稳的 $3 \sim 7 \mathrm{~s}$ 这段时间 增大了 $\beta_{d}$ 的跟踪权重减小 $r_{d}$ 的跟踪权重, 从而 抑制了质心侧偏角的增大, 保证了车辆的稳定 性, 但是以横摆角速度出现较大的跟踪偏差

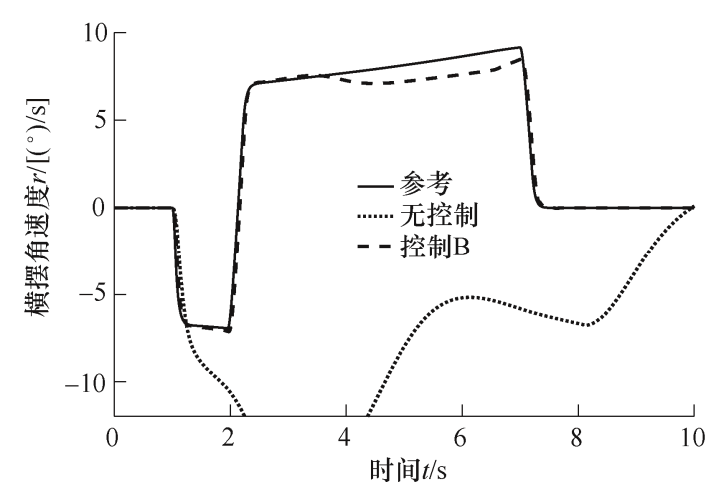

图 25 横摆角速度
(图 25, 3 7 $\mathrm{s}$ 阶段)为代价。从车辆安全性的角 度来看, 这种权衡是合理的。

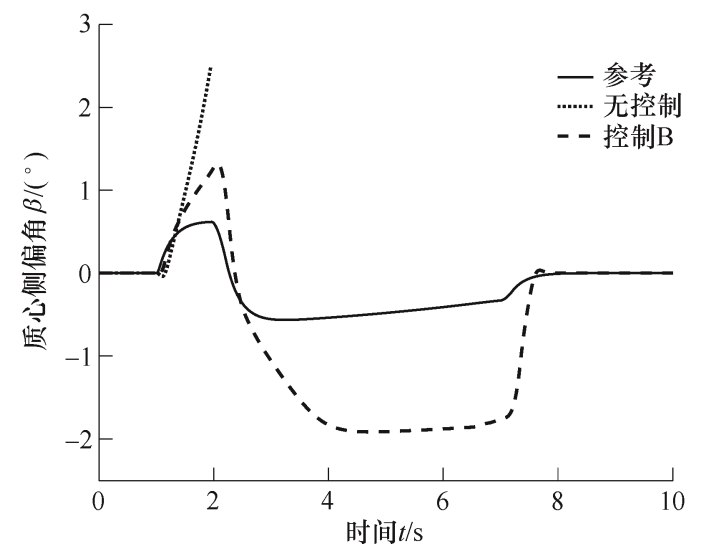

图 26 质心侧偏角

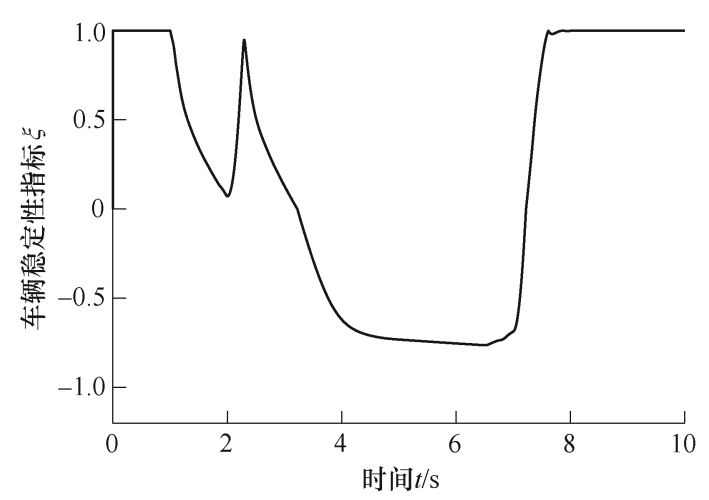

图 27 车辆稳定性指标 $\xi$

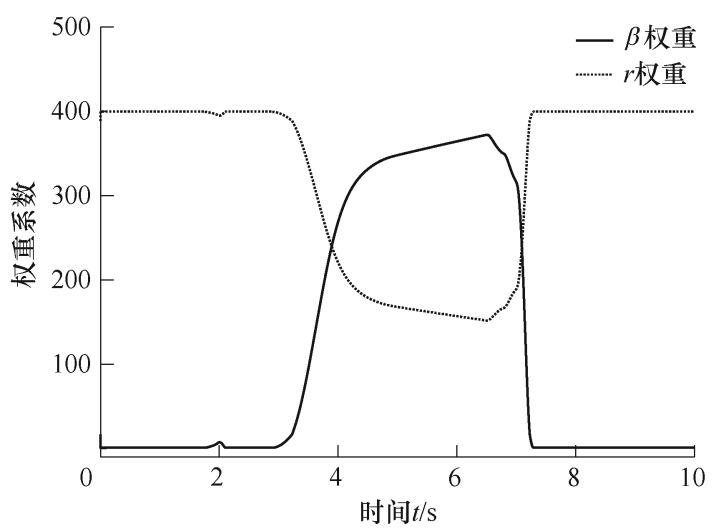

图 28 车辆质心侧偏角与横摆角速度跟踪权重系数

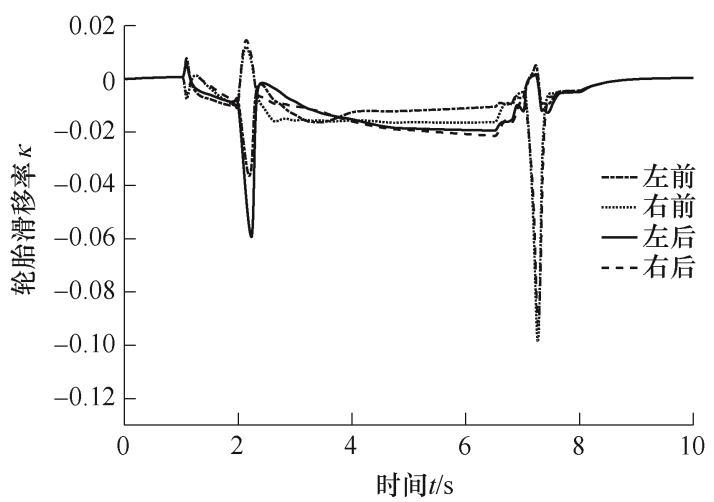

图 29 控制器 B: 轮胎滑移率 


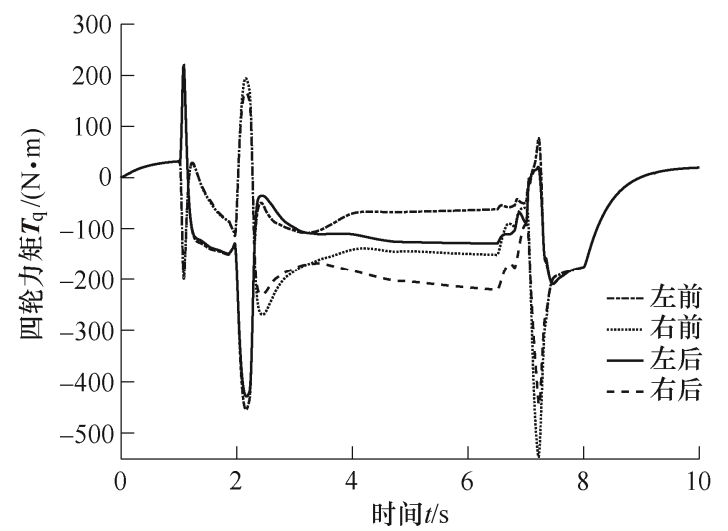

图 30 控制器 $\mathrm{B}$ : 四轮力矩分配

该仿真结果表明了稳定性指标和自适应权重分 配在 MPC 控制器中的有效性, 在极限工况下能够 有效的保证车辆的稳定性。

\section{4 硬件在环验证}

本节通过硬件在环系统 (Hardware in the loop, HiL)对控制算法在实际工程应用中的有效性和实时 性进行了验证。由于 MPC 控制算法需要在每一步 执行优化求解过程, 进行矩阵运算和二次规划 (Quadratic programming, QP)求解, 因而能否在控制 器中实时运行成为 MPC 控制算法在工程应用中需 要考虑的问题 ${ }^{[11]}$ 。为了验证本文提出的 MPC 控制 算法在实际工程应用中的有效性和实时性, 本节采 用 dSPACE 公司的 MicroAutobox II 作为快速原型控 制器运行 MPC 算法。采用 NI-RT 仿真系统运行 CarSim 整车模型作为被控对象(图 31), 此外, 测试 系统采用 CAN 通信的方式, 与实车通信方式一致。 在测试中将 MicroAutobox II 的运行步长设置为 $10 \mathrm{~ms}$, 即算法的更新频率为 $100 \mathrm{~Hz}$; NI-RT 系统的 更新频率为 $1000 \mathrm{~Hz}$; 系统中的 CAN 信号发送频率 都设置为 $100 \mathrm{~Hz}$ 。

由于本节主要验证算法在工程应用中的效果, 因而采用第 3.1 节中介绍的低附蛇形工况作为验证 工况, 设置与第 3.1 节相同。预测步长取 $N_{p}=3$ (本文 控制步长与预测步长相同)。测试结果如图 32 34 所示。可以看到在图 32 中, 实际的横摆角速度准确 地跟踪上了参考值, 只是在第 $2.5 \sim 3.5 \mathrm{~s}$ 出现抖动。 图 33 为四轮力矩分配效果, 复合工况下后轴的两个 车轮峰值力矩明显小于前轴的两个车轮, 表明算法 在实际工程中的应用是有效的。图 28 为轮胎滑移
率曲线, 轮胎滑移率得到了很好的控制, 最大值约 为 0.13 。这三幅图中, 第 $2.5 \sim 3.5 \mathrm{~s}$ 阶段曲线都出 现了抖动, 这是由于快速原型控制器中算法的更新 频率为 $100 \mathrm{~Hz}$, 远小于软件仿真 $1000 \mathrm{~Hz}$ 的频率导 致的。但是抖动并不会发散和持续，因而能够满足 实际工程应用的需求。

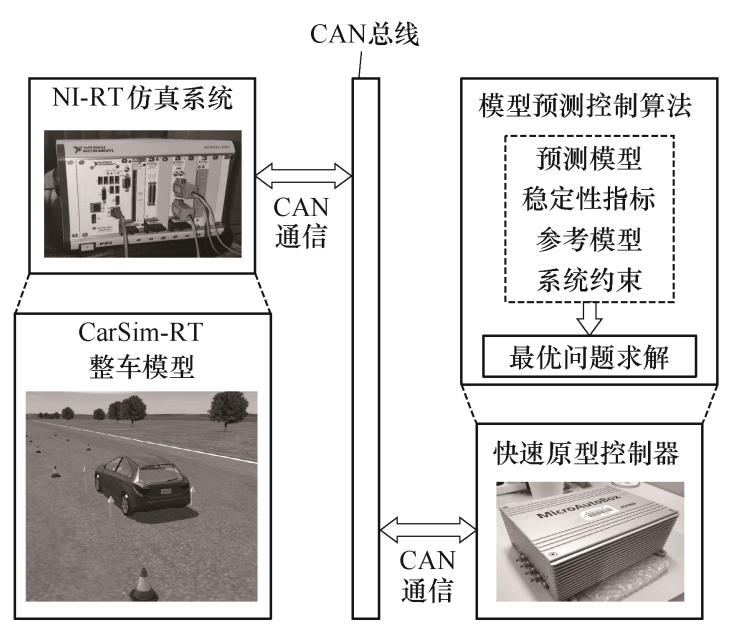

图 31 硬件在环测试

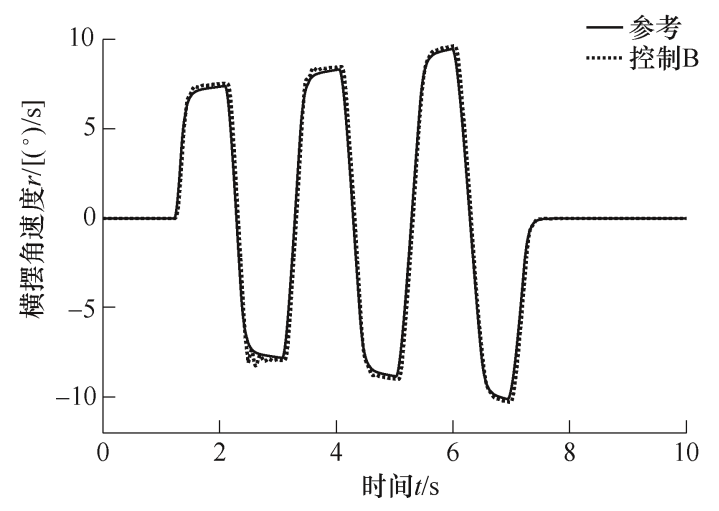

图 $32 \mathrm{HiL}$ 验证: 横摆角速度

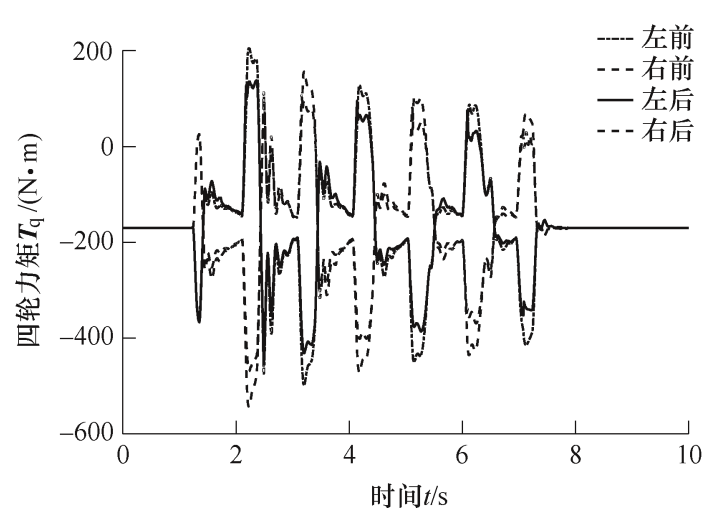

图 $33 \mathrm{HiL}$ 验证: 四轮力矩分配 


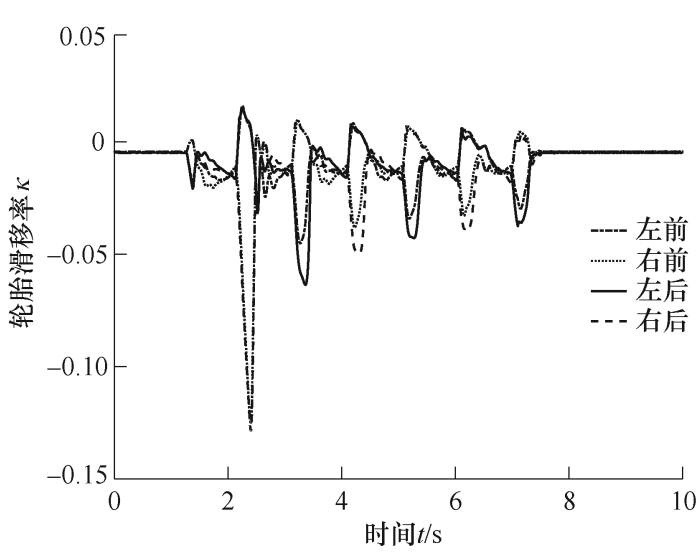

图 34 HiL 验证: 轮胎滑移率

\section{5 结论}

本文设计了一个模型预测控制器(MPC)用于复 合工况下四轮驱动电动汽车的操纵稳定性控制。主 要有如下特点。

(1) 提出了面向控制的复合工况 UniTire 轮胎模 型, 推导出了有效侧偏刚度 $K_{y c}$ 及其导数的解析表 达式, 使其能够应用于控制器的设计。通过在控制 器设计中考虑纵向力(滑移率)对轮胎侧偏特性的影 响, 复合工况下控制器能够更加合理地决策各个车 轮所需的力矩值, 有效地抑制了轮胎滑移率的增大, 降低了电机驱动/制动力矩需求。

(2) 提出了基于轮胎侧偏状态的车辆稳定性评 价指标, 该指标能够定量的描述车辆的稳定性程度, 可以根据稳定性指标(数值大小)判断车辆是处于稳 定、过渡还是失稳区域, 可以为控制器的设计提供 重要的车辆稳定性指标信息。

(3) 采用可变权重的多目标 MPC 控制器结构, 能够根据车辆状态实时调节操纵性、稳定性以及轮 胎滑移率的控制权重。稳定性指标作为重要的输入 信息对操纵性权重和稳定性权重进行调节, 当车辆 状态处于稳定区域时, 以操纵性控制目标为主, 当 车辆状态进入过渡或失稳区域时, 以稳定性控制 为主, 仿真结果表明, 当车辆状态进入过渡区域时, 控制器就发挥作用, 使车辆状态不会进入失稳区域, 如此有利于在保证车辆稳定的同时使车辆也具有更 好的极限操纵性。由于采用双曲函数作为轮胎滑移 率约束方程, 因而只有当轮胎滑移率较大时权重系 数才会快速平滑增大, 有利于减小滑移率约束对控 制系统的影响同时提高滑移率约束效果。

\section{参 考 文 献}

[1] KIHM A, TROMMER S. The new car market for electric vehicles and the potential for fuel substitution[J]. Energy Policy, 2014, 73(1): 147-157.

[2] WANG R, CHEN Y, FENG D. Development and performance characterization of an electric ground vehicle with independently-actuated in-wheel motors[J]. J. Power Sources, 2011, 196(8): 3962-71.

[3] MAEDA K, FUJIMOTO H, HORI Y. Four-wheel driving-force distribution method for instantaneous or split slippery roads for electric vehicle[J]. Automatika, 2013, 54(1): 103-113.

[4] DATA S, FRIGERIO F. Objective evaluation of handling quality $[\mathrm{J}]$. Proc. Institution Mech. Eng. Part D, J. Automobile Eng., 2002, 216(4): 297- 305.

[5] NOVELLIS L, SORNIOTTI A, GRUBER P. Wheel torque distribution criteria for electric vehicles with torque-vectoring differentials[J]. IEEE Transactions on Vehicular Technology, 2014, 63(4): 1593-1602.

[6] LU Q, GENTILE P, TOTA A, et al. Enhancing vehicle cornering limit through sideslip and yaw rate control[J]. Mechanical Systems and Signal Processing, 2015, 75(1): 455-472.

[7] ZHANG L, DING H, HUANG Y, et al. An analytical approach to improve vehicle manoeuvreability via torque vectoring control: Theoretical study and experimental validation[J]. IEEE Transactions on Vehicular Technology, 2019, 68(5): 4514-4526.

[8] ZHAI L, SUN T, WANG J. Electronic stability control based on motor driving and braking torque distribution for a four in-wheel motor drive electric vehicle[J]. IEEE Transactions on Vehicular Technology, 2016, 65(1): 4726-4739.

[9] HAN Z, XU N, CHEN H, et al. Energy-efficient control of electric vehicles based on linear quadratic regulator and phase plane analysis[J]. Applied Energy, 2018，213(1): 639-657.

[10] DI C, TSENG H, BERNARDINI D, et al. Vehicle yaw stability control by coordinated active front steering and differential braking in the tire sideslip angles domain[J]. IEEE Transactions on Control Systems Technology, 2013, 21(4): 1236-1248.

[11] BEAL C, CHRISTIAN G. Model predictive control for vehicle stabilization at the limits of handling[J]. IEEE Transactions on Control Systems Technology, 2012, 21(4): 1258-1269.

[12] BEAL C, BOBIER C G, GERDES J C. Controlling 
vehicle instability through stable handling envelopes[C/CD]//ASME 2011 Dynamic Systems and Control Conference and Bath/ASME Symposium on Fluid Power and Motion Control. American Society of Mechanical Engineers, 2011.

[13] BROWN M, FUNKE J, ERLIEN S, et al. Safe driving envelopes for path tracking in autonomous vehicles[J]. Control Engineering Practice, 2017, 61(1): 307-316.

[14] HAO Z, XIAN L, SHU S, et al. Phase plane analysis for vehicle handling and stability[J]. International Journal of Computational Intelligence Systems, 2011, 4(6) : 1179-1186.

[15] YUAN L, ZHAO H, CHEN H, et al. Nonlinear MPC-based slip control for electric vehicles with vehicle safety constraints $[\mathrm{J}]$. Mechatronics，2016，38(1): 1-15.

[16] JALALI M, HASHEMI E, KHAJEPOUR A, et al. A combined-slip predictive control of vehicle stability with experimental verification[J]. Vehicle System Dynamics, 2018, 56(2): 319-340.

[17] 郭孔辉. UniTire 统一轮胎模型 $[\mathrm{J}]$. 机械工程学报, 2016, 52(12): 90-99.

GUO Konghui. UniTire: Unified tire model[J]. Journal of Mechanical Engineering, 2016， 52(12): 90-99.

[18] ATAEI M, KHAJEPOUR A. Model predictive control for integrated lateral stability, traction/braking control, and rollover prevention of electric vehicles[J]. Vehicle System Dynamics, 2019, 65(6): 4440-4458.

[19] JALALI M, KHAJEPOUR A, CHEN S, et al. Integrated stability and traction control for electric vehicles using model predictive control[J]. Control Eng. Pract., 2016, 54 (1): 256-266.

[20] ATAEI M, KHAJEPOUR A, JEON S. A novel reconfgurable integrated vehicle stability control withomni actuation systems[J]. IEEE Trans. Veh. Technol., 2017, 67(4): 2945-2957.

[21] GUO K, LU D, CHEN S-K, et al. The UniTire model: A nonlinear and non-steady-state tyre model for vehicle dynamics simulation[J]. Vehicle System Dynamics, 2005 43(Suppl.): 341-358.

[22] GUO K, XU N, LU D, et al. A model for combined tire cornering and braking forces with anisotropic tread and carcass stiffness[J]. SAE Int. J. Commer. Veh., 2011, 4(1): 84-95.

[23] 许男. 复合工况下轮胎稳态模型研究 $[D]$. 长春: 吉林大 学, 2012 .
XU Nan. Study on the steady state tire model under combined conditions[D]. Changchun: Jilin University, 2012.

[24] 李小雨, 许男, 仇韬, 等. 各向异性刚度对轮胎力学特 性及车辆操纵性的影响 [J]. 吉林大学学报, 2020 , 50(2): 389-398.

LI Xiaoyu, XU Nan, QIU Tao, et al. Influence of anisotropic stiffness on tire mechanical properties and vehicle handling characteristics under combined slip situations[J]. Journal of Jilin University, 2020，50(2): 389-398

[25] FALCONE P, ERIC H, BORRELLI F, et al. MPC-based yaw and lateral stabilization via active front steering and braking[J]. Vehicle System Dynamics, 2008, 46(Suppl.): 611-628.

[26] HASHEMI E, PIRANI M, KHAJEPOUR A, et al. Corner-based estimation of tire forces and vehicle velocities robust to road conditions[J]. Control Engineering Practice, 2017, 61: 28-40.

[27] MIRZAEI M. A new strategy for minimum usage of external yaw moment in vehicle dynamic control system[J]. Transport. Res. Part C: Emerg. Technol., 2010, 18(2): 213-225

[28] BOBIER C, BEAL C, KEGELMAN J, et al. Vehicle control synthesis using phase portraits of planar dynamics[J]. Vehicle System Dynamics, 2019, 57(9): 1318-1337.

[29] FERREAU H, KIRCHES C, POTSCHKA A, et al. qpOASES : A parametric active-set algorithm for quadratic programming $[\mathrm{J}]$. Math. Program Comput., 2014, 6(4): 327-363.

[30] ZHAO H, GAO B, REN B, et al. Integrated control of in-wheel motor electric vehicles using a triple-step nonlinear method[J]. Journal of the Franklin Institute, 2015, 352(2): 519-540.

[31] YUAN L, ZHAO H, CHEN $\mathrm{H}$, et al. Nonlinear MPC-based slip control for electric vehicles with vehicle safety constraints[J]. Mechatronics，2016，38(1): 1-15.

作者简介：许男，男，1988 年出生，博士，副教授，博士研究生导师。 主要研究方向为轮胎力学、车辆动力学与控制。

E-mail: xunan@jlu.edu.cn

李小雨(通信作者), 男, 1989 年出生, 博士研究生。主要研究方向为车 辆动力学与控制、车辆电子控制技术。

E-mail: lixiaoyuhy@foxmail.com 